\title{
Effect of nanoparticle on growth, biochemical and anatomical characteristics of moringa plant (Moringa oleifera L.) under salinity stress condition
}

\author{
Abou-Shlell M. K. , El-Emary F. A., KHalifa A. A. \\ Agricultural Botany Department, Faculty of Agriculture, Al-Azhar University (Assiut \\ Branch), Assiut, Egypt
}

\begin{abstract}
Two pot experiments were carried out at the Experimental Farm of the Agricultural Botany Department, Faculty of Agriculture, Al-Azhar University (Assiut branch), Assiut, Egypt, during two successive seasons of 2018 and 2019. The main aim of this study was to find out the effect of both of foliar application with some growth promoters (using nanotechnology) i.e., zinc, iron, copper oxide nanoparticles at 50,100 and $150 \mathrm{mgl}^{-1}$ of each and silica nanoparticles at 20,40 and $60 \mathrm{mgl}^{-1}$ as well as salinity stress level of irrigation water at 9000 $\mathrm{mgl}^{-1}$ individually and their interactions on growth, biochemical and anatomical characteristics of Moringa oliefera L. plant. The results showed that individually salinity stress level of irrigation water at $9000 \mathrm{mgl}^{-1}$ decreased all studied vegetative growth parameters of moringa plant i.e., plant height $(\mathrm{cm})$, stem diameter $(\mathrm{cm})$, leaves number plant ${ }^{-1}$, leaf area plant ${ }^{-1}$ as well as root, stem and leaves dry weights plant ${ }^{-1}$ compared with the control. The same trend was obtained in photosynthetic pigments content (total chlorophyll) as well as the studied anatomical characteristics of root section (i.e., root diameter of V.C and length of xylem arch), stem section (i.e., stem diameter, $\varnothing$ of V.C, length of V.B in the stem) and Leaf blade section (i.e., upper epidermis thickness, lower epidermis thickness and mesophyll tissue for leaflet) at 50 days of Moringa oliefera leaves compared with unstressed plant. On other hand, salinity stress level at $9000 \mathrm{mgl}^{-1}$ resulted in increasing antioxidant activity as well as total phenolic contents at 60 days of Moringa oliefera leaves compared with unstressed plant. Data also indicated that the individually foliar application treatments with Zinc, Iron, Copper oxide nanoparticles at 50,100, 150 of each and Silica at 20, 40, $60 \mathrm{mgl}^{-1}$ nanoparticles increased studied vegetative growth as well as anatomical characteristics, chemical compositions, photosynthetic pigments (total chlorophyll), antioxidants activity and total phenolic contents of moringa plant compared with the control treatment. In this respect, Silica nanoparticles at $60 \mathrm{mgl}^{-1}$ followed by zinc and iron oxide at $150 \mathrm{mgl}^{-1}$ nanoparticles of each were the most effective treatments, respectively. Finally, Based on these results, it could be concluded that the foliar application with nanoparticles gave the highest Valois of morphological, chemical compositions and anatomical characteristics its that reduce the harmful effects of salinity stress of Moringa oliefera L. plant specially at silica $60 \mathrm{mgl}^{-1}$,. Data recorded that this results using it under the same field conditions (Assiut governorate, Egypt).
\end{abstract}

Keywords: Moringa oleifera, nanoparticles, salinity, growth, bioconstituents, anatomy.

*Corresponding author: Abou-Shlell M. K.,

E-mail address: mohamedkhames660@yahoo.com 


\section{Introduction}

Moringa (Moringa oleifera $\mathrm{L}$.) is the most widely cultivated species of Moringace family due to its easy propagation, fast growth and its numerous economic uses. Moringa oleifera L. in the botanical scientific taxonomy belong to Kingdom: Plantae, Division: Magnoliphyta, Class: agnoliopsida, Order: Brassicales, Family: Moringaceae, Genus: Moringa, Species: M.oleifera (Fahey, 2005). Moringa oleifera L. is one of the most useful tropical trees, its leaves are extremely valuable source of nutrition for people of all ages. Nutritional analysis indicates that moringa leaves contain affluence of essential disease preventing nutrients. They even contain all of the essential amino acids which are unusual for a plant source. The young leaves are edible and are commonly cooked and eaten like spinach or used for making soups and salads. It is an exceptionally good source of antioxidant compounds such as flavonoids, ascorbic acid, carotenoids phenolics and some mineral nutrients (in particularly iron) and the sulphurcontaining amino acids methionine as well as cystine. The composition of amino acids in the leaf protein is well balanced; they contain high amounts of many of these nutrients and total phenols also a very low source of fat (Abdull Razis et al., 2014; Mishra et al., 2012; Osman and Abohassan, 2012). Soil salinity threshold levels depend on a crop species, variety, developmental stage and environmental factors. One of the most important a biotic stress factors is soil salinity. It causes great effects on growth development, yield of crops (Chaparzadeh et al., 2004) and causes great losses in crops yield (Smirnoff, 1998). Seed germination also affected by the excessive content of salt in soil solution, particularly in case of sensitive plants. Regarding, the interaction between plants and salinity stress, there are several mechanisms: (1) selective ions accumulation/exclusion (2) control of ion uptake by roots and their transport into leaves (3) prevention of $\mathrm{Na}^{+}$ and $\mathrm{Cl}^{-}$accumulation in the cytoplasm (4) synthesis and accumulation of nontoxic (compatible) osmolytes in the cytosol (5) change in photosynthetic pathway (6) induction of antioxidative system (7) stimulation of phytohormones production, such as abscisic and jasmonic acids. All these mechanisms are realized at the levels of whole plant and plant tissues as well as the cell molecular level (Dajic, 2006). The effect of salinity on root (An et al., 2003) and leaf anatomy ( $\mathrm{Hu}$ and Schmidhalter, 2001; Kiliç et al., 2007) of plants had already been reported in previous works. Many researchers reported that with an increasing salinity there was a decreasing in xylem tissue development. Pimmongkol et al. (2002) stated that width and diameter of the vascular bundles of rice stem decreased in $\mathrm{NaCl}$ medium. Junghans et al. (2006) showed that high salt concentrations reduced the cambial activity in Populus euphratica. Salinity causes total leaf area reducing (Awang et al., 1993) as well as leaf thickness increasing (Raafat et al., 1991). It is also reducing the vascular tissues development (Belda and Ho, 1993). It increases trichome density and decreases or has no effect on stomatal density (Ludders and Kaminski, 1991). Salinity affects ion accumulation in 
leaves, thereby membrane permeability and chlorophyll synthesis. Micronutrients are playing important roles in crop yield increasing. Micronutrients have prominent effects on dry matter accumulation and yield of plants (Asad and Rafique, 2000). As for Iron, it plays roles in biological redox system, enzyme activation and oxygen carrier in nitrogen fixation (Romheld and Marschner, 1991). Zinc is important to membrane integrity and phytochrome activities (Shkoinik, 1984). Also Zinc plays an important role in the plant production, and chlorophyll synthesis (Kaya and Higgs, 2002). It can reduce the negative effects of ROS in environmental stresses and its deficiency decreases plant resistance (Cakmak and Marschner, 1988; McCauley et al., 2009). $\mathrm{Cu}$ is one of the most essential elements in chlorophyll synthesis, enhance plant photosynthesis and carbohydrate metabolism, help to regulate water movement through plant and play vital role in seed production, in addition to it is vital for physiological redox processes, pollen viability and lignification as well as increasing yield components (Marschner, 1995). Therefore, much attention is needed for adequate and balanced use of micronutrients along with macronutrients to enhance the response to organic fertilizers (Baddaruddin et al., 1999). Silicon ( $\mathrm{Si}$ ) is an environmental friendly and ecologically compatible for stimulating plant growth. It was reported that silicon plays a role in reducing the hazard effects of several a biotic and biotic stresses such as drought stress (Etesamy and Jeong, 2018; Laing and Adandonon, 2005). It has emerged as an important mineral for many horticultural crops (Ma, 2004). It is contributing elasticity of the cell wall during extension growth. It is interacting with cell constituents as polyphenols and pectins and this increasing elasticity of the cell wall. Also, increasing of silicon absorption led for maintaining erect leaves and important for leaf angle to photosynthesis (Emadian and Newton, 1989). Foliar spray with silicon significantly increased yield and its components of pea plant (Gharib and Hanafy, 2005). Foliar application with potassium silicate $\left(\mathrm{KSiO}_{3}\right)$ increased growth of sunflower plant (Kamenidou and Cavins, 2008). Sayed et al. (2018) found that globe artichoke plant sprayed with silicon at $2000 \mathrm{mgl}^{-1}$ recorded the highest increasing in all studied characteristics i.e., growth aspects, chlorophylls content, nitrogen, phosphorus, potassium, total sugars and total amino acids concentrations as well as the yield parameters compared with untreated plant. Remero Aranda et al. (2006) reported that, $\mathrm{Si}$ is improving the storage of water within plant tissues, which allows a higher rate of growth. Abd El-Aal et al. (2019) confirmed that spraying taro plant grown under water stress levels with potassium silicate at $2500 \mathrm{mgl}^{-1}$ improved plant tolerability to the harmful effects of water stress. Hence, the present study was conducted to evaluate the effects of different salinity level of irrigation water and foliar spray with some stimulant nutrients nanoparticle i.e., zinc, iron, copper and silica treatments individually and the combination between the foliar application treatments within the salinity level on vegetative growth parameters, 
chemical compositions and anatomical characteristics of moringa plant for studying the possibility for improving plant tolerability to the harmful effects of salinity stress conditions.

\section{Materials and methods}

Two pot experiments were carried out at the experimental farm of the agricultural botany department, faculty of agriculture, Al-Azhar university, Assiut branch, Egypt during the two successive seasons of 2018 and 2019 to investigate the effect of foliar spray with some nanoparticles and salinity level of irrigation water individually as well as the combination between the foliar application treatments within salinity level $9000 \mathrm{mgl}^{-1}$ on vegetative growth characteristics, chemical constituents at 60 days and anatomical characteristics of Moringa oliefera plant at 50 days. Seeds of moringa (Moringa oleifera L.) were obtained from National Research Center, Dokki, Giza, Egypt. While, zinc, iron, copper oxide and Silica nanoparticles were obtained from Nano technology Company (3 Ibn-Eyas. St., Roxy, Heliopolis, Cairo). Moringa seeds were sowed at $1^{\text {th }}$ March for 2018 and 2019 seasons in plastic pots of $30 \mathrm{~cm}$ in diameter which filled with $6 \mathrm{~kg}$ with a mixture of 1 clay: 1 sand :1 peat moss (v:v:v), then seeds were sowed (2-3 seeds / pot). After emergences of seedlings, were thinned to one plant per pot, this experiment included 26 treatments, each treatment was about 3 replications (4 pots for each replicate). The pots were arranged in split plot design. Cultivation and all cultural practices except irrigation i.e., weeding, fertilization and pest control and so on were performed according to the recommendations as usual. Foliar application with nanoparticle treatments were carried out four times, first at 30 days and repeated every 15 days as foliar spray to cover completely the plant foliage.

\subsection{The experiment treatments}

This experiment included 26 treatments, which were the foliar application with some nanoparticle and salinity level individually treatments as well as their interactions.

\subsubsection{Salinity level of irrigation water}

$\mathrm{NaCl}$ was used to apply the level of salinity stress, salinity level of irrigation water were used in addition to the control (tap water) i.e., $9000 \mathrm{mgl}^{-1}$ as well as the control of water requirements of moringa plants in both seasons. All the experimental units were received equal amounts of water until complete germination at 30 days, then applying of water irrigation salinity treatment were started in both seasons.

\subsubsection{Application of nanoparticle treatments}

Some nutrients nanoparticle individually 
treatments i.e., zinc, iron, copper oxide nanoparticles at 50,100 and $150 \mathrm{mgl}^{-1}$ of each and silica nanoparticles at 20, 40 and $60 \mathrm{mgl}^{-1}$ in addition to the control (tap water) were used as foliar applications. Plants were sprayed with the foliar application treatments four times after 30, 45, 60 and 75 days.

\subsection{Sampling and collecting data}

\subsubsection{Growth characteristics}

Different morphological characteristics of moringa plant were measured and calculated at 60 days. Three plants from each treatment were randomly taken and then separated into their organs and the following characteristics were recorded: plant height $(\mathrm{cm})$, stem diameter $(\mathrm{cm})$, leaves number plant ${ }^{-1}$, leaf area plant ${ }^{-1}$, root as well as stem and leaves dry weight plant $^{-1}$. The samples were dried in the oven-dried for $48 \mathrm{~h}$ in $75^{\circ} \mathrm{C}$ to a constant weight and then the dry weight per plant was calculated. These dry samples were kept in for chemical analysis.

\subsubsection{Physiological characteristics}

Chemical analyses were carried out in the leaves sample at 60 days during both seasons of 2018 and 2019. Total chlorophyll was calorimetrically determined in the fresh leaves according to the method described by Sadasivam and Manickam (1997). The antioxidants activity was determined in dry leaves according to the method by Saint-Cricq De Gaulejae et al. (1999) modified by Lu et al. (2007). Total phenolic compounds content was determined according to Folin-Ciocalteu spectrophotometric method (Lu et al., 2007).

\subsubsection{Anatomical study}

Specimens of moringa (Moringa oleifera L.) root, stem and terminal leaflet were collected in the second season only. The root samples were taken $0.5 \mathrm{~cm}$ from the root tip and the $5^{\text {th }}$ apical internode of the main stem and its corresponding leaf of treated plants with salinity level i.e., $9000 \mathrm{mgl}^{-1}$ and applied nanoparticle i.e., zinc, iron, copper at 150 of each $\mathrm{mgl}^{-1}$ as well as silica at $60 \mathrm{mgl}^{-1}$ and their interaction in addition to those of the control at 50 days. The specimens were taken then killed and fixed in FAA (5 ml. formalin, $15 \mathrm{ml}$. glacial acetic acid and $90 \mathrm{ml}$. ethyl alchohol $70 \%$, distilled water $35 \mathrm{ml}$ ), washed in 50\% ethyl alcohol, dehydrated in series of ethyl alchohols $70,80,90,95$ and $100 \%$, infiltrated in xylene, embedded in paraffin wax with a melting point of $40-45^{\circ} \mathrm{C}$, sectioned on a rotary microtome at a thickness of 5-7 $\mu \mathrm{m}$., stained with the double method (light green and safranin), cleared in xylene and mounted in Canada balsam (Johanson, 1940) .Sections were read to detect histological manifestation of noticeable responses resulted from treatments. The prepared sections were microscopically examined; counts and measurements $(\mu)$ were taken by 
computerized morphometrical analysis, the morphometrical analysis was done by research microscope type Axiostar plus made by Zeiss transmitted light bright field examinations upgrade able to professional digital image analysis system (Carl Zeiss Axiovision Product Suite DVD 30).

\subsubsection{Statistical analysis}

Statistical analysis conducted using Costat computer statistical software package, Data were statistically analyzed according to the analysis of variance (ANOVA) of the completely randomized design, applied in both laboratory and greenhouse experiments according to Gomez and Gomez (1984). Least Significant Difference (LSD) test at 5\% was used to determine genotypic differences among all means of pathological, morphological and anatomical traits under each treatment.

\section{Results and Discussion}

\subsection{Vegetative growth characteristics}

Data presented in Table (1a, b) show the effect of the individually treatment of irrigation water salinity level i.e., 9000 $\mathrm{mgl}^{-1}$ and applied nutrients nanoparticle i.e., zinc, iron, copper and silica as well as the effect of the applied nanoparticles on the vegetative growth characteristics i.e., plant height $(\mathrm{cm})$, stem diameter $(\mathrm{cm})$, leaves number plant $^{-1}$, leaf area plant $^{-1}$, root, stem and leaves dry weight plant $^{-1}$ of moringa plant grown under salinity stress level $9000 \mathrm{mgl}^{-1}$ at 60 days during the two growing seasons of 2018 and 2019.

\subsubsection{Effect of salinity stress}

All pot experiments under this study indicated that salinity level of irrigation water at $9000 \mathrm{mgl}^{-1}$ have decreased all studied vegetative growth characteristics of moringa plant. The same data also cleared that the level of salinity i.e., 9000 $\mathrm{mgl}^{-1}$ was the most effective treatment in this respect when compared with unstressed plant (the control) at 60 days during the two experimental seasons. Plants growing under saline conditions are stressed basically in three ways; (1) reduced water potential in the root zone causing water deficit, (2) phytotoxicity of ions such as $\mathrm{Na}^{+}$and $\mathrm{Cl}^{-}$, and (3) nutrients imbalance by uptake depression and shoot transport (Marschner, 1995). Salinity became a basic problem when sufficient salts accumulate in the root zone to negatively affect plant growth. Excess salts in the root zone prevent plant roots from withdrawing water from the surrounding soil. This lowers the amount of water available to the plant, regardless of the amount of water actually in the root zone (Abdelhamid $e t$ al., 2010). The effect of salinity on plant growth is related to the stage of plant development at which salinity is imposed (Ayres and Westcot, 1985). The reduction in most vegetative growth parameters may be caused by the 
reduction in the cell size which might be attributed to changes in osmotic cell enlargement dependent on solute accumulation (Asin et al., 2007) or due to drastic changes in ion relationship (Grossmann et al., 1994). High salinity causes both hyper-ionic and hyperosmotic stress and can lead to plant demise (Wilson et al., 2006). Moreover, salt treatment affects differently early growth stages of plants and has both osmotic and specific ion effects on plant growth (Dionisio-Sese and Tobita, 2000). Moreover, the reduction in growth is generally the consequences of several physiological responses including modication of ion balance, water status, mineral nutrition, stomatal behavior, photosynthetic efficiency and carbon allocation and utilization (Greenway and Munns, 1980). In salt-sensitive plant, shoot and root growth is permanently reduced within hours of salt stress and this does not appear to depend on $\mathrm{Na}^{+}$ concentrations in the growing tissues, but rather is a response to the osmolatity of the external solution (Munns, 2003).The decrease in growth due to salinity may be attributed to an increase in respiration rate resulting from higher energy requirements (Sakr et al., 2013). These results may attributed to the effect of salinity stress on the water content of the leaves, as suggested by $\mathrm{Hu}$ et al. (2007). High level of salinity negatively affected shoot dry weight. Salinity can damage the plant through its osmotic effect, which is equivalent to a decrease in water activity through specific toxic effects of ions and by disturbing the uptake of essential nutrients (Dorais et al., 2001; Gomaa et al., 2008).

Table (1a): Effect of salinity stress level, applied nanoparticle and their interaction treatments on moringa growth parameters at 60 days during 2018 and 2019 seasons.

\begin{tabular}{|c|c|c|c|c|c|c|c|c|c|c|c|c|c|c|c|}
\hline \multirow[b]{2}{*}{ Treatments } & \multirow[b]{2}{*}{$\begin{array}{l}\text { Con. } \\
\mathrm{mgl}^{-1}\end{array}$} & \multicolumn{7}{|c|}{2018} & \multicolumn{7}{|c|}{2019} \\
\hline & & $\begin{array}{c}\text { Plant } \\
\text { height } \\
(\mathrm{cm})\end{array}$ & $\begin{array}{c}\text { Stem } \\
\text { diameter } \\
(\mathrm{cm})\end{array}$ & $\begin{array}{l}\text { Leaves } \\
\text { number } \\
\text { plant- }^{-1}\end{array}$ & $\begin{array}{c}\text { Leaf } \\
\text { area } \\
\mathrm{Cm}^{2} \\
\text { plant }^{-1} \\
\end{array}$ & \begin{tabular}{|c|} 
Root \\
dry \\
weight $g$ \\
plant $^{-1}$
\end{tabular} & \begin{tabular}{|c|}
$\begin{array}{c}\text { Stem } \\
\text { dry } \\
\text { weight } g \\
\text { plant }^{-1}\end{array}$ \\
\end{tabular} & \begin{tabular}{|c|}
$\begin{array}{c}\text { Leaves } \\
\text { dry } \\
\text { weight } \\
\text { g plant }^{-1}\end{array}$ \\
\end{tabular} & $\begin{array}{l}\text { Plant } \\
\text { height } \\
(\mathrm{cm})\end{array}$ & $\begin{array}{c}\text { Stem } \\
\text { diameter } \\
(\mathrm{cm})\end{array}$ & $\begin{array}{c}\text { Leaves } \\
\text { number } \\
\text { plant }^{-1}\end{array}$ & $\begin{array}{l}\text { Leaf area } \\
\mathrm{cm}^{2} \text { plant }^{-1}\end{array}$ & \begin{tabular}{|c|}
$\begin{array}{c}\text { Root } \\
\text { dry } \\
\text { weight } \\
\text { plant }^{-1}\end{array}$ \\
\end{tabular} & $\begin{array}{l}\text { Stem dry } \\
\text { weight } g \\
\text { plant }^{-1}\end{array}$ & $\begin{array}{l}\text { Leaves dry } \\
\text { eight g plant }\end{array}$ \\
\hline \multicolumn{16}{|c|}{ Effect of salinity } \\
\hline Tap water & - & 47.89 & 2.00 & 30.33 & 168.12 & 2.06 & 2.35 & 3.52 & 50.97 & 2.20 & 32.28 & 173.31 & 2.48 & 2.77 & 4.03 \\
\hline Salinity & 9000 & 30.51 & 1.18 & 16.82 & 103.58 & 1.07 & 1.35 & 2.09 & 32.36 & 1.38 & 18.82 & 108.82 & 1.49 & 1.77 & 2.49 \\
\hline L.S.D & 0.05 & \begin{tabular}{|l|}
1.33 \\
\end{tabular} & 0.26 & 1.02 & 3.22 & 0.24 & 0.27 & 1.00 & 2.44 & 0.28 & 2.33 & 1.99 & 0.33 & 0.29 & 0.23 \\
\hline \multicolumn{16}{|c|}{ Effect of applied nanoparticles } \\
\hline Control & - & \begin{tabular}{|l|}
26.67 \\
\end{tabular} & 1.00 & 14.50 & 91.69 & 1.06 & 1.23 & 1.80 & 30.17 & 1.20 & 16.50 & 97.49 & 1.38 & 1.63 & 2.20 \\
\hline \multirow{3}{*}{$\mathrm{Zn}$} & 50 & 37.83 & 1.45 & 22.83 & 125.43 & 1.41 & 1.69 & 2.57 & 40.33 & 1.65 & 24.83 & 130.66 & 1.85 & 2.17 & 3.04 \\
\hline & 100 & 41.83 & 1.62 & 26.33 & 144.77 & 1.63 & 1.93 & 2.93 & 44.33 & 1.87 & 28.33 & 149.96 & 2.06 & 2.40 & 3.39 \\
\hline & 150 & 46.33 & 1.82 & 29.83 & 162.17 & 1.89 & 2.17 & 3.30 & 48.83 & 2.02 & 31.83 & 167.28 & 2.27 & 2.64 & 3.75 \\
\hline \multirow{3}{*}{$\mathrm{Fe}$} & 50 & 36.33 & 1.52 & 21.33 & 120.97 & 1.36 & 1.63 & 2.49 & 38.33 & 1.72 & 23.33 & 126.23 & 1.81 & 2.09 & 2.93 \\
\hline & 100 & \begin{tabular}{|l|}
40.33 \\
\end{tabular} & 1.73 & 24.33 & 140.16 & 1.57 & 1.87 & 2.84 & 42.33 & 1.93 & 26.33 & 145.34 & 2.00 & 2.34 & 3.30 \\
\hline & 150 & \begin{tabular}{|l|}
44.83 \\
\end{tabular} & 1.93 & 27.83 & 157.72 & 1.80 & 2.11 & 3.20 & 46.83 & 2.13 & 29.83 & 163.06 & 2.22 & 2.58 & 3.64 \\
\hline \multirow{3}{*}{$\mathrm{Cu}$} & 50 & 34.83 & 1.37 & 19.83 & 116.64 & 1.31 & 1.57 & 2.37 & 36.83 & 1.57 & 21.83 & 121.81 & 1.75 & 2.03 & 2.82 \\
\hline & 100 & \begin{tabular}{|l|}
38.83 \\
\end{tabular} & 1.55 & 22.83 & 135.65 & 1.52 & 1.81 & 2.77 & 40.83 & 1.75 & 24.50 & 141.01 & 1.95 & 2.28 & 3.23 \\
\hline & 150 & \begin{tabular}{|l|}
43.33 \\
\end{tabular} & 1.77 & 26.33 & 153.19 & 1.73 & 2.05 & 3.11 & 45.33 & 1.97 & 28.33 & 158.34 & 2.16 & 2.53 & 3.56 \\
\hline \multirow{3}{*}{$\mathrm{Si}$} & 20 & \begin{tabular}{|l|}
35.33 \\
\end{tabular} & 1.43 & 20.33 & 119.98 & 1.46 & 1.75 & 2.67 & 38.33 & 1.63 & 22.33 & 124.98 & 1.90 & 2.24 & 3.13 \\
\hline & 40 & \begin{tabular}{|l|}
39.33 \\
\end{tabular} & 1.65 & 23.33 & 141.13 & 1.69 & 1.99 & 3.02 & 42.33 & 1.85 & 25.33 & 146.13 & 2.12 & 2.48 & 3.48 \\
\hline & 60 & 43.83 & 1.87 & 26.83 & 156.57 & 2.00 & 2.23 & 3.42 & 46.83 & 2.07 & 28.83 & 161.57 & 2.33 & 2.72 & 3.90 \\
\hline \multicolumn{2}{|l|}{ L.S.D 0.05} & \begin{tabular}{|l|}
0.88 \\
\end{tabular} & 0.08 & 0.57 & 2.02 & 0.05 & 0.06 & 0.07 & 0.99 & 0.14 & 0.27 & 1.34 & 0.19 & 0.08 & 0.12 \\
\hline
\end{tabular}


Table (1b): Effect of salinity stress level, applied nanoparticle and their interaction treatments on moringa growth parameters at 60 days during 2018 and 2019 seasons.

\begin{tabular}{|c|c|c|c|c|c|c|c|c|c|c|c|c|c|c|c|c|}
\hline \multirow{2}{*}{\multicolumn{2}{|c|}{ Treatments }} & \multirow[b]{2}{*}{$\begin{array}{l}\text { Con. } \\
\mathrm{mgl}^{-1}\end{array}$} & \multicolumn{7}{|c|}{2018} & \multicolumn{7}{|c|}{2019} \\
\hline & & & $\begin{array}{c}\begin{array}{c}\text { Plant } \\
\text { height } \\
(\mathrm{cm})\end{array} \\
\end{array}$ & $\begin{array}{c}\text { Stem } \\
\text { diameter } \\
(\mathrm{cm})\end{array}$ & $\begin{array}{c}\text { Leaves } \\
\text { number } \\
\text { plant- }^{1}\end{array}$ & $\begin{array}{c}\text { Leaf } \\
\text { area } \\
\mathrm{Cm}^{2} \\
\text { plant }^{-1} \\
\end{array}$ & \begin{tabular}{|c|}
$\begin{array}{c}\text { Root } \\
\text { dry } \\
\text { weight } g \\
\text { plant }^{-1}\end{array}$ \\
\end{tabular} & \begin{tabular}{c|c}
$\begin{array}{c}\text { Stem } \\
\text { dry } \\
\text { weight } g \\
\text { plant }^{-1}\end{array}$ \\
\end{tabular} & $\begin{array}{c}\text { Leaves } \\
\text { dry } \\
\text { weight } \\
\text { g plant }^{-1} \\
\end{array}$ & $\begin{array}{l}\text { Plant } \\
\text { height } \\
(\mathrm{cm})\end{array}$ & $\begin{array}{c}\text { Stem } \\
\text { diameter } \\
(\mathrm{cm})\end{array}$ & $\begin{array}{c}\text { Leaves } \\
\text { number } \\
\text { plant- }^{1}\end{array}$ & $\begin{array}{l}\text { Leaf area } \\
\mathrm{cm}^{2} \text { plant }^{-1}\end{array}$ & \multirow[t]{2}{*}{\begin{tabular}{|c|} 
Root \\
dry \\
weight $g$ \\
plant $^{-1}$
\end{tabular} \mid} & \multirow[t]{2}{*}{$\begin{array}{l}\text { Stem dry } \\
\text { weight g } \\
\text { plant }^{-1}\end{array}$} & \multirow[t]{2}{*}{$\begin{array}{l}\text { eaves d } \\
\text { veight } \mathrm{g} \\
\text { plant }^{-1}\end{array}$} \\
\hline & & & & & & & Effe & ct of appli & ed nanop & rticles & & & & & & \\
\hline \multirow{13}{*}{ 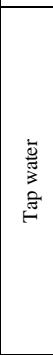 } & Control & - & 38.67 & 1.60 & 24.33 & 137.20 & 1.52 & 1.82 & 2.78 & 42.67 & 1.80 & 26.33 & 142.30 & 1.90 & 2.29 & 3.23 \\
\hline & \multirow{3}{*}{$\mathrm{Zn}$} & 50 & \begin{tabular}{|l|}
47.33 \\
\end{tabular} & 1.90 & 31.33 & 159.26 & 1.90 & 2.18 & 3.22 & 50.33 & 2.10 & 33.33 & 164.66 & 2.34 & 2.66 & 3.75 \\
\hline & & 100 & \begin{tabular}{|l|}
51.33 \\
\end{tabular} & 2.07 & 34.33 & 177.33 & 2.12 & 2.42 & 3.62 & 54.33 & 2.27 & 36.33 & 182.59 & 2.56 & 2.89 & 4.14 \\
\hline & & 150 & \begin{tabular}{|l|}
56.33 \\
\end{tabular} & 2.27 & 37.33 & 196.35 & 2.42 & 2.66 & 4.04 & 59.33 & 2.47 & 39.33 & 201.31 & 2.76 & 3.13 & 4.56 \\
\hline & \multirow{3}{*}{$\mathrm{Fe}$} & 50 & 45.33 & 1.93 & 29.33 & 154.30 & 1.84 & 2.12 & 3.12 & 48.33 & 2.13 & 31.33 & 159.77 & 2.29 & 2.58 & 3.63 \\
\hline & & 100 & \begin{tabular}{|l|}
49.33 \\
\end{tabular} & 2.13 & 31.33 & 173.13 & 2.06 & 2.36 & 3.52 & 52.33 & 2.33 & 33.33 & 178.67 & 2.50 & 2.83 & 4.03 \\
\hline & & 150 & \begin{tabular}{|l|}
54.33 \\
\end{tabular} & 2.33 & 34.33 & 191.44 & 2.30 & 2.60 & 3.93 & 57.33 & 2.53 & 36.33 & 196.69 & 2.71 & 3.07 & 4.44 \\
\hline & \multirow{3}{*}{$\mathrm{Cu}$} & 50 & 43.33 & 1.77 & 27.33 & 149.61 & 1.78 & 2.06 & 3.00 & 46.33 & 1.97 & 29.33 & 154.76 & 2.22 & 2.51 & 3.51 \\
\hline & & 100 & \begin{tabular}{|l|}
47.33 \\
\end{tabular} & 1.97 & 29.33 & 169.45 & 2.02 & 2.30 & 3.44 & 50.33 & 2.17 & 30.67 & 174.60 & 2.45 & 2.77 & 3.95 \\
\hline & & 150 & 52.33 & 2.20 & 32.33 & 186.51 & 2.22 & 2.54 & 3.82 & 55.33 & 2.40 & 34.33 & 191.66 & 2.65 & 3.02 & 4.33 \\
\hline & \multirow{3}{*}{$\mathrm{Si}$} & 20 & 41.33 & 1.73 & 25.33 & 144.33 & 1.96 & 2.24 & 3.33 & 44.33 & 1.93 & 27.33 & 149.33 & 2.40 & 2.51 & 3.84 \\
\hline & & 40 & \begin{tabular}{|l|}
45.33 \\
\end{tabular} & 1.93 & 27.33 & 165.33 & 2.18 & 2.48 & 3.73 & 48.33 & 2.13 & 29.33 & 170.33 & 2.62 & 2.77 & 4.24 \\
\hline & & 60 & \begin{tabular}{|l|}
50.33 \\
\end{tabular} & 2.17 & 30.33 & 181.36 & 2.57 & 2.72 & 4.16 & 53.33 & 2.37 & 32.33 & 186.36 & 2.82 & 3.02 & 4.70 \\
\hline \multirow{13}{*}{ 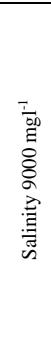 } & Control & - & \begin{tabular}{|l|}
14.67 \\
\end{tabular} & 0.40 & 4.67 & 46.19 & 0.602 & 0.642 & 0.822 & 17.67 & 0.60 & 6.67 & 52.69 & 0.863 & 0.973 & 1.17 \\
\hline & \multirow{3}{*}{$\mathrm{Zn}$} & 50 & 28.33 & 1.00 & 14.33 & 91.59 & 0.922 & 1.20 & 1.92 & 30.33 & 1.20 & 16.33 & 96.66 & 1.36 & 1.67 & 2.32 \\
\hline & & 100 & 32.33 & 1.17 & 18.33 & 112.22 & 1.14 & 1.44 & 2.24 & 34.33 & 1.37 & 20.33 & 117.33 & 1.55 & 1.91 & 2.63 \\
\hline & & 150 & 36.33 & 1.37 & 22.33 & 127.98 & 1.36 & 1.68 & 2.55 & 38.33 & 1.57 & 24.33 & 133.24 & 1.78 & 2.15 & 2.93 \\
\hline & \multirow{3}{*}{$\mathrm{Fe}$} & 50 & 27.33 & 1.10 & 13.33 & 87.64 & 0.88 & 1.14 & 1.85 & 28.33 & 1.30 & 15.33 & 92.68 & 1.32 & 1.60 & 2.22 \\
\hline & & 100 & 31.33 & 1.33 & 17.33 & 107.19 & 1.08 & 1.38 & 2.16 & 32.33 & 1.53 & 19.33 & 112.01 & 1.49 & 1.86 & 2.57 \\
\hline & & 150 & \begin{tabular}{|l|}
35.33 \\
\end{tabular} & 1.53 & 21.33 & 124.00 & 1.30 & 1.62 & 2.46 & 36.33 & 1.73 & 23.33 & 129.42 & 1.72 & 2.09 & 2.84 \\
\hline & & 50 & \begin{tabular}{|l|}
26.33 \\
\end{tabular} & 0.967 & 12.33 & 83.67 & 0.84 & 1.08 & 1.74 & 27.33 & 1.17 & 14.33 & 88.86 & 1.28 & 1.55 & 2.12 \\
\hline & & 100 & 30.33 & 1.13 & 16.33 & 101.86 & 1.02 & 1.32 & 2.09 & 31.33 & 1.33 & 18.33 & 107.43 & 1.44 & 1.79 & 2.50 \\
\hline & $\mathrm{Cu}$ & 150 & \begin{tabular}{|l|}
34.33 \\
\end{tabular} & 1.33 & 20.33 & 119.86 & 1.24 & 1.56 & 2.39 & 35.33 & 1.53 & 22.33 & 125.01 & 1.66 & 2.04 & 2.79 \\
\hline & & 20 & \begin{tabular}{|l|}
29.33 \\
\end{tabular} & 1.13 & 15.33 & 95.64 & 0.962 & 1.26 & 2.00 & 32.33 & 1.33 & 17.33 & 100.64 & 1.40 & 1.55 & 2.42 \\
\hline & $\mathrm{Si}$ & 40 & 33.33 & 1.37 & 19.33 & 116.92 & 1.20 & 1.50 & 2.31 & 36.33 & 1.57 & 21.33 & 121.92 & 1.62 & 1.79 & 2.71 \\
\hline & S1 & 60 & 37.33 & 1.57 & 23.33 & 131.78 & 1.42 & 1.74 & 2.67 & 40.33 & 1.77 & 25.33 & 136.78 & 1.84 & 2.04 & 3.09 \\
\hline L.S.D & 0.05 & & \begin{tabular}{|l|}
0.98 \\
\end{tabular} & 0.10 & 0.49 & 1.33 & 0.018 & 0.06 & 0.011 & 0.81 & 0.07 & 0.42 & 2.22 & 0.09 & 0.010 & 0.012 \\
\hline
\end{tabular}

The ability of the plant response to saline stress can be hardly explained by the fact that salinity imposes both an ionic and osmotic stress, which causes reduction in roots growth and weights (Pasternak, 1987). More in these respect, salinity was found to reduce shoot and root weights as well as photosynthates reduction (Brugnoli and Lauteri, 1991). Moreover, the reduction in shoot and root dry weight accumulation might be attributed to a decrease in either leaf number and leaf area, decreasing in $\mathrm{Co}_{2}$ uptake in leaves mainly because $\mathrm{Na} \mathrm{Cl}$ treatment, decrease stomatal conductance and consequently less $\mathrm{Co}_{2}$ is available for carboxylation reaction in the photosynthesis apparatus (Sakr et al., 2013).

\subsubsection{Effect of applied nanoparticles treatments}

In both seasons of study, all foliar application with nanoparticle treatments increased root, stem and leaves dry weight as well as total leaf area when compared to the untreated plant (the control) at 60 days. In this respect, the solely treatments of zinc, iron, copper oxide at $150 \mathrm{mgl}^{-1}$ of each as well as silica nanoparticles at $60 \mathrm{mgl}^{-1}$ recorded the highest values of vegetative growth parameters of moringa plant. Moreover, 
obtained data cleared that foliar spraying treatments have important roles in alleviating and mitigating the harmful effects of salinity stress when compared with the control. Our results go on line with those reported that application of zinc and iron resulted in useful changes in growth attributes. The possible cause for such positive role is enhancing in the activity of bio-substances or activity of photosynthetic system (Mekkdad, 2017; Quary et al., 2006) or might be due to the active role of these trace-elements in metabolic processes of plants and photosynthesis and thus, reflected to increase the growth attributes. Abou ElNasr et al. (2015) found that foliar sprays with iron nanoparticles increased stem diameter, leaf area and dry weights. Prasad et al. (2012) found that zinc oxide nanoparticles increased plant growth parameters. Sedghi et al. (2013) found that zinc oxide nanoparticles increased plant growth and development. Therefore, it could be concluded that increasing leaf area as well as increment of dry matter accumulation Table (1a, b) as well as photosynthetic pigments Table (2) in leaves of moringa plants reverse the stimulating effect of these treatments on the photosynthetic efficiency process, thereby more photosynthates being created as well as enhancing minerals translocation from root to leaves.

\subsubsection{Effect of the interactions (salinity $x$ nanoparticles)}

With regarding to the interaction treatments i.e., the effect of foliar application treatments with nanoparticles on vegetative growth characteristics of moringa plant grown under the level of used salinity stress i.e., $9000 \mathrm{mgl}^{-1}$, the data indicated that the individually foliar spray treatments of zinc, iron, copper at $150 \mathrm{mgl}^{-1}$ of each and silica nanoparticles at $60 \mathrm{mgl}^{-1}$ significantly enhanced growth attributes of moringa plant under salinity stress level at $9000 \mathrm{mgl}^{-1}$ which were the most effective treatments when compared with other treatments and the control during the two growing seasons. In this regard, the foliar application treatments with nanoparticles basically mitigate the harmful effect of salinity stress on growth attributes. Silica at $60 \mathrm{mgl}^{-1}$ followed by zinc, iron and copper oxide nanoparticles at $150 \mathrm{mgl}^{-1}$ of each respectively, were the most effective in this respect.

\subsection{Bioconstituents content}

\subsubsection{Photosynthetic pigments content}

Data presented in Table (2) show the effect of irrigation water salinity level at $9000 \mathrm{mgl}^{-1}$ and applied nutrients nanoparticle i.e., zinc, iron, copper and silica in addition to the effect of the applied nanoparticle treatments on photosynthetic pigments content (total chlorophyll content $\mathrm{mg} \mathrm{g}^{-1}$ f.wt.) in leaves of moringa plant grown under salinity stress level at $9000 \mathrm{mgl}^{-1}$ at 60 days during the two growing seasons of 2018 and 2019. 
Table (2): Effect of salinity stress level, applied nanoparticle and interaction treatments on some bioconstituents of moringa leaves at 60 days during 2018 and 2019 seasons.

\begin{tabular}{|c|c|c|c|c|c|c|c|c|}
\hline \multirow{2}{*}{\multicolumn{2}{|c|}{ Treatments }} & \multirow[b]{2}{*}{$\begin{array}{l}\text { Con. } \\
\mathrm{mgl}^{-1}\end{array}$} & \multicolumn{3}{|c|}{2018} & \multicolumn{3}{|c|}{2019} \\
\hline & & & $\begin{array}{l}\text { Total chl. } \\
\mathrm{Mg} \mathrm{g}^{-1} \text { f.wt }\end{array}$ & $\begin{array}{l}\text { Antioxidants } \\
\text { activity } \\
\mathrm{Mg} \mathrm{g}^{-1} \text { d.wt. }\end{array}$ & $\begin{array}{l}\text { Total phenolic } \\
\mathrm{mg} \mathrm{g}^{-1} \text { d.wt. }\end{array}$ & $\begin{array}{l}\text { Total chl. } \\
\mathrm{Mg} \mathrm{g}^{-1} \text { f.wt }\end{array}$ & $\begin{array}{l}\text { Antioxidants } \\
\text { activity } \\
\mathrm{Mg} \mathrm{g}^{-1} \text { d.wt. }\end{array}$ & $\begin{array}{l}\text { Total phenolic } \\
\mathrm{mg} \mathrm{g}^{-1} \text { d.wt. }\end{array}$ \\
\hline \multicolumn{9}{|c|}{ Effect of salinity } \\
\hline \multicolumn{2}{|c|}{ Tap water } & - & 2.145 & 67.93 & 19.63 & 1.986 & 69.04 & 20.75 \\
\hline \multicolumn{2}{|c|}{ Salinity } & 9000 & 1.337 & 60.43 & 17.01 & 1.360 & 61.49 & 18.11 \\
\hline \multicolumn{3}{|c|}{ L.S.D 0.05} & 0.304 & 1.11 & 1.05 & 0.313 & 1.22 & 1.04 \\
\hline \multicolumn{9}{|c|}{ Effect of applied nanoparticles } \\
\hline \multicolumn{2}{|l|}{ Control } & - & 1.284 & 60.26 & 13.45 & 1.309 & 61.11 & 14.57 \\
\hline \multirow{3}{*}{\multicolumn{2}{|c|}{$\mathrm{Zn}$}} & 50 & 1.512 & 62.26 & 17.70 & 1.535 & 63.36 & 18.80 \\
\hline & & 100 & 1.773 & 64.26 & 18.63 & 1.796 & 65.36 & 19.73 \\
\hline & & 150 & 2.048 & 66.26 & 19.51 & 2.074 & 67.36 & 20.61 \\
\hline \multirow{3}{*}{\multicolumn{2}{|c|}{$\mathrm{Fe}$}} & 50 & 1.577 & 63.01 & 18.06 & 1.602 & 64.11 & 19.16 \\
\hline & & 100 & 1.840 & 65.01 & 18.98 & 1.862 & 66.11 & 20.08 \\
\hline & & 150 & 2.108 & 67.01 & 19.83 & 2.132 & 68.11 & 20.93 \\
\hline \multirow{3}{*}{\multicolumn{2}{|c|}{$\mathrm{Cu}$}} & 50 & 1.467 & 62.51 & 17.83 & 1.490 & 63.61 & 18.93 \\
\hline & & 100 & 1.679 & 64.51 & 18.70 & 1.731 & 65.61 & 19.80 \\
\hline & & 150 & 1.977 & 66.51 & 19.66 & 1.997 & 67.61 & 20.76 \\
\hline \multirow{3}{*}{\multicolumn{2}{|c|}{$\mathrm{Si}$}} & 20 & 1.550 & 62.26 & 17.68 & 1.574 & 63.36 & 18.78 \\
\hline & & 40 & 1.781 & 64.27 & 18.69 & 1.804 & 65.37 & 19.86 \\
\hline & & 60 & 2.034 & 66.26 & 19.51 & 2.057 & 67.36 & 20.61 \\
\hline \multicolumn{3}{|c|}{ L.S.D 0.05} & 0.007 & 0.88 & 0.62 & 0.022 & 0.45 & 0.33 \\
\hline \multicolumn{9}{|c|}{ Effect of interactions } \\
\hline \multirow{13}{*}{ 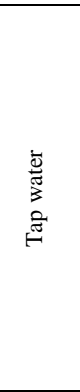 } & Control & - & 1.831 & 64.01 & 11.88 & 1.857 & 65.21 & 13.02 \\
\hline & \multirow{3}{*}{$\mathrm{Zn}$} & 50 & 1.960 & 66.00 & 19.25 & 1.982 & 67.10 & 20.35 \\
\hline & & 100 & 2.203 & 68.02 & 20.20 & 2.226 & 69.12 & 21.30 \\
\hline & & 150 & 2.455 & 70.00 & 21.00 & 2.479 & 71.10 & 22.10 \\
\hline & \multirow{3}{*}{$\mathrm{Fe}$} & 50 & 2.010 & 66.50 & 19.51 & 2.038 & 67.60 & 20.61 \\
\hline & & 100 & 2.257 & 68.52 & 20.40 & 2.279 & 69.62 & 21.50 \\
\hline & & 150 & 2.514 & 70.50 & 21.25 & 2.537 & 71.60 & 22.35 \\
\hline & \multirow{3}{*}{$\mathrm{Cu}$} & 50 & 1.928 & 67.00 & 19.75 & 1.950 & 68.10 & 20.85 \\
\hline & & 100 & 2.068 & 69.02 & 20.60 & 2.149 & 70.12 & 21.70 \\
\hline & & 150 & 2.400 & 71.00 & 21.50 & 2.419 & 72.10 & 22.60 \\
\hline & & 20 & 1.876 & 65.51 & 19.00 & 1.899 & 66.61 & 20.10 \\
\hline & $\mathrm{Si}$ & 40 & 2.068 & 67.52 & 20.08 & 2.091 & 68.62 & 21.31 \\
\hline & $S_{1}$ & 60 & 2.313 & 69.50 & 20.80 & 2.336 & 70.60 & 21.90 \\
\hline & Control & - & 0.737 & 56.51 & 15.01 & 0.762 & 57.02 & 16.11 \\
\hline & & 50 & 1.065 & 58.51 & 16.15 & 1.088 & 59.61 & 17.25 \\
\hline & $\mathrm{Zn}$ & 100 & 1.343 & 60.50 & 17.05 & 1.367 & 61.60 & 18.15 \\
\hline & & 150 & 1.640 & 62.52 & 18.01 & 1.668 & 63.62 & 19.11 \\
\hline Б0 & & 50 & 1.144 & 59.51 & 16.60 & 1.166 & 60.61 & 17.70 \\
\hline 8 & $\mathrm{Fe}$ & 100 & 1.423 & 61.50 & 17.55 & 1.446 & 62.60 & 18.65 \\
\hline ஓু & & 150 & 1.702 & 63.52 & 18.40 & 1.726 & 64.62 & 19.50 \\
\hline तે & & 50 & 1.007 & 58.01 & 15.91 & 1.030 & 59.11 & 17.01 \\
\hline$\Xi$ & $C_{1}$ & 100 & 1.290 & 60.01 & 16.80 & 1.314 & 61.11 & 17.90 \\
\hline$\tilde{\omega}$ & $\mathrm{Cu}$ & 150 & 1.553 & 62.02 & 17.81 & 1.575 & 63.12 & 18.91 \\
\hline & & 20 & 1.225 & 59.01 & 16.35 & 1.248 & 60.11 & 17.45 \\
\hline & $\mathrm{Si}$ & 40 & 1.495 & 61.02 & 17.30 & 1.517 & 62.12 & 18.40 \\
\hline & S1 & 60 & 1.755 & 63.01 & 18.21 & 1.778 & 64.11 & 19.31 \\
\hline L.S.D C & & & 0.022 & 0.53 & 0.15 & 0.010 & 0.48 & 0.09 \\
\hline
\end{tabular}

\subsubsection{Effect of salinity stress}

Regarding to the effect of irrigation water salinity level, obtained data clearly indicate that stressed plants grown under salinity stress level recorded lowest values of total chlorophyll content when compared with the control. In other 
words, salinity level of irrigation water i.e., $\quad 9000 \mathrm{mgl}^{-1}$ decreased total chlorophyll content of moringa leaves compared with the control. In this respect, salinity level at $9000 \mathrm{mgl}^{-1}$ treatment recorded the highest reduction in the content of total chlorophyll. Increasing of sodium concentration in plant tissues could be resulted in increasing the oxidative stress, which causes deterioration in chloroplast structure and an associate losing in chlorophyll, this leads to a decrease in chlorophyll content, while carotenoids increased (Khosravinejad and Faboondia, 2008). Increasing salinity under stress conditions significantly reducing photosynthesis by closure of the stomata, in which decreasing carbon dioxide supply and then growth is reducing (Jonas et al., 1992). During water stress produced by salt stress, production of reactive oxygen species (ROS) and reduction of chloroplast stromal volume are also thought to play an essential role in inhibiting and limiting photosynthesis (Price and Hendry, 1991). ROS causes chlorophyll deterioration and membrane lipid peroxidation. So, accumulation of lipid peroxidation and chlorophyll retention are two oxidative stress indicators (Yildirim et al., 2008).

\subsubsection{Effect of applied nanoparticle treatments}

Concerning the effect of stimulants foliar spray, the same data in Table (2) also, clear that all applied nanoparticle treatments recorded significant increases of photosynthetic pigments compared with untreated plant. In this regard, iron followed by zinc, copper oxide at 150 $\mathrm{mgl}^{-1}$ of each and silica nanoparticles at $60 \mathrm{mgl}^{-1}$ recorded the highest values compared with the other treatments and control.

\subsubsection{Effect of the interaction}

As for the effect of interaction, data clearly showed that the foliar application nanoparticle treatments led for enhancing the photosynthetic pigments content of moringa leaves grown under salinity stress level at $9000 \mathrm{mgl}^{-1}$ compared with untreated plant. Applied nanoparticle treatments basically mitigate the harmful effect of salinity stress on photosynthetic pigments. Silica at $60 \mathrm{mgl}^{-1}$ followed by Iron, Zinc and Copper oxide nanoparticles at $150 \mathrm{mgl}^{-1}$ of each respectively, were the most effective treatments in this respect.

\subsubsection{Antioxidants activity and total phenolic content}

Data presented in Tables (2) indicate the effect of irrigation water salinity level at $9000 \mathrm{mgl}^{-1}$ and applied nutrients nanoparticle i.e., zinc, iron, copper and silica furthermore the effect of applied nanoparticle treatments on antioxidants activity as well as total phenolic content (mg g ${ }^{-1}$ D.wt.) in leaves of moringa plant grown under salinity stress level 9000 $\mathrm{mgl}^{-1}$ at 60 days during the two growing 
seasons of 2018 and 2019.

\subsubsection{Effect of salinity stress}

Regarding the effect of irrigation water salinity level, obtained results clearly show that stressed plants grown under salinity stress level at $9000 \mathrm{mgl}^{-1}$ recorded highest values of antioxidants activity and total phenolic content when compared with the control. Moreover, salinity level of irrigation water increased antioxidants activity and total phenolic content of moringa leaves compared with the control. Highest salt concentration normally impair the cellular electron transport within the different sub-cellular compartments and lead to the generation of reactive oxygen species (ROS) such as singlet oxygen, superoxide, hydrogen peroxide and hydroxyl radicals. ROS led for enhancing antioxidants system defense by increasing the accumulation of non-enzymatic antioxidants such as phenolic compounds, vitamin $\mathrm{C}$ and tocopherol (Bellaire et al., 2000; Mckersie et al., 1996; Tanaka et al., 1994).

\subsubsection{Effect of applied nanoparticle treatments}

Concerning the effect of stimulants foliar spray, the same data in Table (2) also, clear that all applied nanoparticle treatments recorded significant increases of antioxidants activity and total phenolic content compared with untreated plant. In this regard, copper followed by iron, zinc oxide at $150 \mathrm{mgl}^{-1}$ of each and silica nanoparticles at $60 \mathrm{mgl}^{-1}$ recorded the highest values compared with the other treatments and control.

\subsubsection{Effect of the interaction}

With regarding to the effect of interaction, results clearly show that the foliar application nanoparticle treatments led for enhancing the antioxidants activity and total phenolic content of moringa leaves grown under salinity stress level at $9000 \mathrm{mgl}^{-1}$ compared with untreated plant. Applied nanoparticle treatments basically mitigate the harmful effect of salinity stress on antioxidants activity and total phenolic content. iron at $150 \mathrm{mgl}^{-1}$ followed by silica at $60 \mathrm{mgl}^{-1}$, zinc and copper oxide nanoparticles at $150 \mathrm{mgl}^{-1}$ of each respectively, were the most effective treatments in this respect. In general, increasing antioxidants activity and total phenolic content with different applied treatments considered as a direct result of increasing both photosynthesis rate and efficiency. Also, that was preceded with large photosynthetic area Table (1a, b) and high concentration of photosynthetic pigments (Table 2) under the application of foliar spray with some stimulant nutrients nanoparticle in moringa plant grown under salinity stress level 9000 $\mathrm{mgl}^{-1}$.

\subsubsection{Anatomical study}

The aim of this study was to follow up 
the internal structure characteristics of moringa (Moringa oleifera $\mathrm{L}$.) root, stem and leaflet which exhibited the most noticeable response to salinity stress level at $9000 \mathrm{mgl}^{-1}$ and nutrients nanoparticle foliar application (i.e., zinc, iron and copper oxide nanoparticles at $150 \mathrm{mgl}^{-1}$ of each as well as silica nanoparticles at $60 \mathrm{mgl}^{-1}$ ) treatments compared with the control. Individually or in combinations on the mean counts and measurements in micron $(\mu)$ at 50 days as a step towards understanding the effect of salinity stress, foliar spray with nutrients nanoparticle and their interactions on moringa internal morphology characteristics based on transverse sections.

\subsubsection{Effect of applied treatments on} anatomical characteristics of moringa root

\subsection{Response to Salinity stress}

Obtained results in Table (3) as well as Figure (1) showed that salinity stress level treatment at $9000 \mathrm{mgl}^{-1}$ decreased the most of moringa root anatomical features compared with the control. Obvious reductions were recorded in the thickness of many anatomical features with salinity stress at $9000 \mathrm{mgl}^{-1}$. Among these anatomical features were the most important ones i.e., root diameter, diameter of vascular cylinder and xylem arch length which were greatly decreased with salinity treatment compared with the control. In other words, control of moringa plant manifested the best results as it exceeded that of salinity level at $9000 \mathrm{mgl}^{-1}$ in terms of most studied anatomical features. In this respect, Neumann (1995) reported that salinity reduces root length and diameter anatomically, it affects cell division and expansion processes. Also, Reinhardt and Rost (1995) as some morphological parameters were negatively affected by salinity; some anatomical variables were diminished as well. Cortical parenchyma and vascular cylinder were reduced. Younis et al. (2014) found that salinity showed a subtractive effect on root anatomy. The statistical data indicate that with the increase in salinity level, there was a significant decrease in root xylem, phloem, cortex and epidermis Akram et al. (2002). Increasing salt levels in roots caused reduction in epidermal area due to cell injuries. Halophytic or salt tolerant species generally possess thick epidermis and this serves as an effective mechanism against water loss during limited moisture availability, but at high salinity levels thickness of epidermis decreased Botti et al. (1998) however, in the salt tolerant species, epidermis thickness greatly increased, which showed its better adaptability because thick epidermis is a specific character of the salt tolerant species (Awasthi and Pathak, 1999; Curtis and Lauchli, 1987; and Kanwal et al., 2012; Nawaz et al., 2006; 2012a,b). 
Table (3): Effect of salinity stress level, applied nanoparticle and interaction treatments on mean counts and measurements of certain histological features of moringa root, stem and leaflet at 50 days during 2019 seasons.

\begin{tabular}{|c|c|c|c|c|c|c|c|c|c|c|c|}
\hline \multicolumn{2}{|c|}{$\begin{array}{c}\text { Treatments } \\
\text { Histological } \\
\text { characteristics } \\
(\mu)\end{array}$} & \multicolumn{3}{|c|}{ Root } & \multicolumn{3}{|c|}{ stem } & \multicolumn{4}{|c|}{ leaflet } \\
\hline \multicolumn{12}{|c|}{ Effect of salinity } \\
\hline \multicolumn{2}{|c|}{ Tap water } & 1760.72 & 1313.08 & 534.74 & 1194.08 & 815.66 & 310.4 & 12.17 & 13.23 & 88.96 & 110.17 \\
\hline \multicolumn{2}{|c|}{ Salinity $9000 \mathrm{mgl}^{-1}$} & 1397.88 & 1001.92 & 373.72 & 1045.46 & 688.16 & 227.1 & 7.84 & 10.74 & 62.97 & 72.36 \\
\hline \multicolumn{12}{|c|}{ Effect of applied nanoparticles } \\
\hline \multicolumn{2}{|c|}{ Control } & 1397.2 & 975.5 & 378.15 & 1060.0 & 687.75 & 224.45 & 7.98 & 10.20 & 61.16 & 77.58 \\
\hline \multicolumn{2}{|c|}{$\mathrm{Zn} 150 \mathrm{mgl}^{-1}$} & 1755.3 & 1351.1 & 490.2 & 1155.0 & 836.8 & 292.7 & 11.96 & 13.04 & 84.79 & 106.5 \\
\hline \multicolumn{2}{|c|}{$\mathrm{Fe} 150 \mathrm{mgl}^{-1}$} & 1610.3 & 1213.4 & 481.7 & 1188.8 & 756.6 & 289.5 & 10.77 & 12.51 & 78.66 & 96.3 \\
\hline \multicolumn{2}{|c|}{$\mathrm{Cu} 150 \mathrm{mgl}^{-1}$} & 1497.9 & 1079.3 & 431.5 & 1080.7 & 735.3 & 271.7 & 9.61 & 11.40 & 76.45 & 85.5 \\
\hline \multicolumn{2}{|c|}{ Si $60 \mathrm{mgl}^{-1}$} & 1635.9 & 1168.2 & 489.7 & 1114.4 & 743.2 & 265.5 & 9.71 & 12.77 & 78.79 & 90.4 \\
\hline \multirow{5}{*}{ 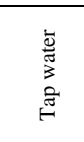 } & Control & 1620.3 & 1183.5 & 467.3 & 1125.0 & 739.0 & 265.6 & 9.38 & 12.62 & 72.66 & 91.06 \\
\hline & $\mathrm{Zn} 150 \mathrm{mgl}^{-1}$ & 2041.3 & 1591.7 & 582.7 & 1258.0 & 968.8 & 347.0 & 15.41 & 14.00 & 98.53 & 132.1 \\
\hline & $\mathrm{Fe} 150 \mathrm{mgl}^{-1}$ & 1754.7 & 1332.7 & 566.0 & 1298.3 & 813.7 & 345.7 & 13.62 & 13.65 & 94.90 & 126.1 \\
\hline & $\mathrm{Cu} 150 \mathrm{mgl}^{-1}$ & 1695.8 & 1250.8 & 532.7 & 1156.3 & 795.8 & 314.0 & 12.21 & 12.94 & 93.05 & 105.5 \\
\hline & Si $60 \mathrm{mgl}^{-1}$ & 1691.5 & 1206.7 & 525.0 & 1132.8 & 761.0 & 279.7 & 10.25 & 12.94 & 85.67 & 96.1 \\
\hline \multirow{5}{*}{ 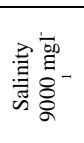 } & Control & 1174.0 & 767.5 & 289.0 & 995.00 & 636.5 & 183.3 & 6.58 & 7.78 & 49.66 & 64.11 \\
\hline & $\mathrm{Zn} 150 \mathrm{mgl}^{-1}$ & 1469.3 & 1110.5 & 397.7 & 1052.0 & 704.8 & 238.3 & 8.51 & 12.09 & 71.04 & 80.9 \\
\hline & Fe $150 \mathrm{mgl}^{-1}$ & 1465.8 & 1094.2 & 397.3 & 1079.3 & 699.5 & 233.3 & 7.93 & 11.37 & 62.41 & 66.4 \\
\hline & $\mathrm{Cu} 150 \mathrm{mgl}^{-1}$ & 1300.0 & 907.7 & 330.3 & 1005.0 & 674.7 & 229.3 & 7.02 & 9.86 & 59.84 & 65.6 \\
\hline & Si $60 \mathrm{mgl}^{-1}$ & 1580.3 & 1129.7 & 454.3 & 1096.0 & 725.3 & 251.3 & 9.16 & 12.60 & 71.91 & 84.8 \\
\hline \multicolumn{2}{|c|}{ L.S.D. $0.05 \%$} & 142.06 & 106.63 & 128.24 & 64.540 & 75.758 & 43.729 & 4.3058 & 3.4602 & 22.81 & 8.7502 \\
\hline
\end{tabular}

$\varnothing=.0 .5$ Diameter of root and stem, $(X=12.5=1 \mathrm{~mm}$ and Leaf $=X=100=200 \mathrm{~mm})$, V.C. $=$ vascular cylinder and V.B.= vascular bundle.

\subsection{Response to foliar application nanoparticle treatments}

Same data in Table (3) and Figure (1) obviously indicate the effect of applied nutrients nanoparticle i.e., zinc, iron and copper oxide nanoparticles at $150 \mathrm{mgl}^{-1}$ of each as well as silica nanoparticles at $60 \mathrm{mgl}^{-1}$ individually treatments on different anatomical features of moringa root. In this respect, most of these applied treatments have positively impacts on the studied histological characteristics of Moringa oleifera L. root i.e., root diameter, diameter of vascular cylinder and xylem arch length when compared with the control. Data also clearly indicate that foliar applications with nutrient nanoparticle of zinc, iron, copper oxide nanoparticles at $150 \mathrm{mgl}^{-1}$ of each and silica nanoparticles at $60 \mathrm{mgl}^{-1}$ recorded highly values of the root studied anatomical characteristics compared with the control treatment. In general, the stimulatory effects of applied treatments upon the anatomy features of treated plants could be attributed to the effect upon cambium activity. Increment of cambium activity could mainly attributed to the increase of endogenous hormones level especially cytokinins and auxins (Abd El-Aal and Eid, 2017; 
Sotiropoulos et al., 2002).

\subsection{Response to salinity stress and applied nanoparticle interaction}

As shown in Table (3) as well as Figure
(2) The obtained data indicate the effect of different applied nutrient nanoparticle treatments upon alleviating the adverse effects of salinity stress on different studied anatomical features of Moringa oleifera $\mathrm{L}$. root.

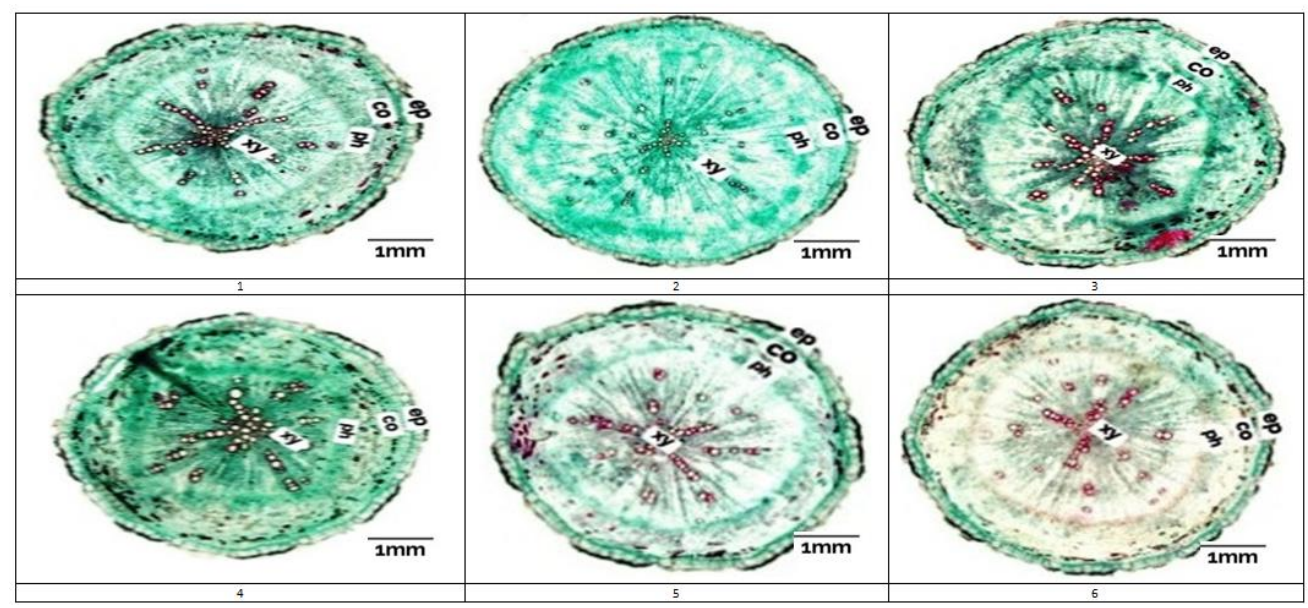

Figure (1): Transverse sections (X 12.5) of moringa root at 50 days as affected by different applied treatments. Where: (1): Control, (2): Salinity, $9000 \mathrm{mgl}^{-1}$, (3): Zinc $150 \mathrm{mgl}^{-1}$, (4): Iron $150 \mathrm{mgl}^{-1}$, (5): Copper $150 \mathrm{mgl}^{-1},(6)$ : Silica $60 \mathrm{mgl}^{-1}$, ep= epidermis , co= cortex tissue, $\mathrm{ph}=$ phloem tissue, $\mathrm{xy}=$ xylem tissue.

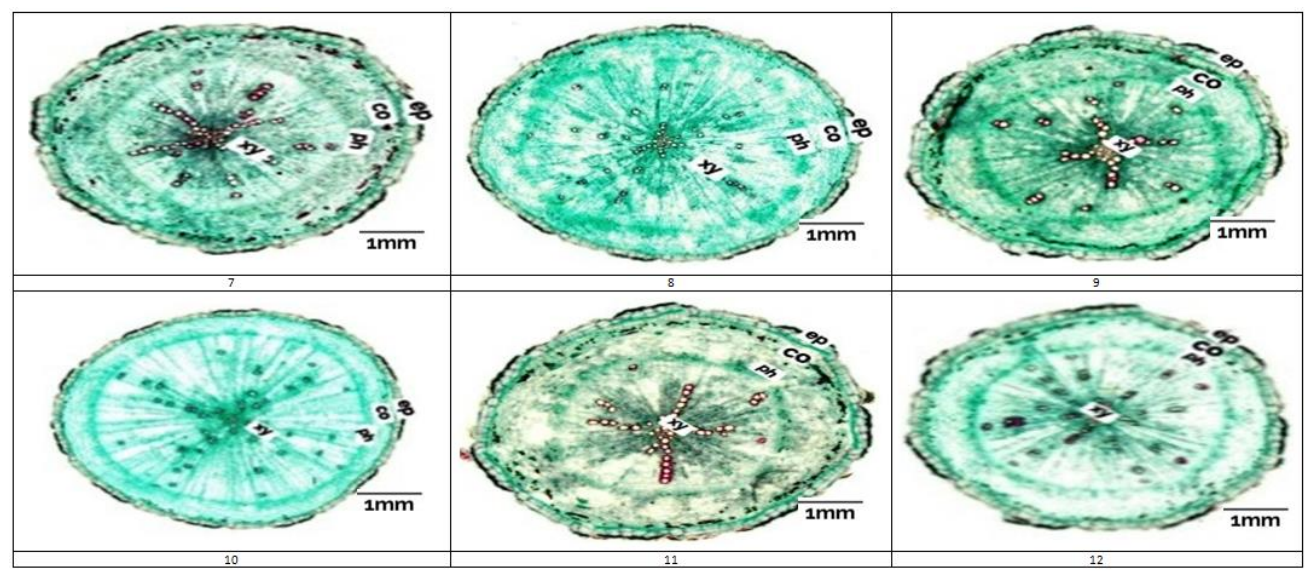

Figure (2): Transverse sections (X 12.5) of moringa root at 50 days as affected by different applied treatments. Where: (7): Control, (8): Salinity $9000 \mathrm{mgl}^{-1}$, (9): Salinity with zinc $150 \mathrm{mgl}^{-1}$, (10): Salinity with iron $150 \mathrm{mgl}^{-1}$, (11): Salinity with copper $150 \mathrm{mgl}^{-1}$, (12): Salinity with silica $60 \mathrm{mgl}^{-1}$, $\mathrm{ep}=$ epidermis, $\mathrm{co}=$ cortex tissue, $\mathrm{ph}=$ phloem tissue, $\mathrm{xy}=\mathrm{xylem}$ tissue. 
In this respect, most of the applied treatments have a positively impact on most histological characteristics of moringa root i.e., diameter of both of root and vascular cylinder as well as length of xylem arch under used salinity stress level at $9000 \mathrm{mgl}^{-1}$ compared with the untreated plant. Also, obtained results clearly show that Moringa oleifera L. plant irrigated with salinity at $9000 \mathrm{mgl}^{-1}$ in combination with Silica nanoparticles at $60 \mathrm{mgl}^{-1}$ followed by zinc and iron oxide nanoparticles at $150 \mathrm{mgl}^{-1}$ of each treatments appeared to be the most effective treatments upon alleviating the adverse effects of the highest salinity stress. Generally, the obtained results clearly indicate that different applied nutrient nanoparticle treatments play a defensive protective role against adverse effects of highest salinity stress level via its regulatory functions.

\subsubsection{Effect of applied treatments on} anatomical characteristics of moringa stem

\subsection{Response to Salinity stress}

As shown in Table (3) and Figure (3) Data indicate the effect of salinity stress at $9000 \mathrm{mgl}^{-1}$ treatment compared with the control upon different anatomical features of moringa stem. In this respect, most of the applied treatments have a negatively impact on most studied histological characteristics of Moringa oleifera L. stem i.e., stem diameter and $\varnothing$ of V.C. as well as length of the V.B. at
50 days. The obtained results show that, the treatment of salinity stress decreased most of moringa stem anatomical features compared with control. Obvious reduction was recorded in the thickness of many anatomical features. Among these anatomical features were the most important ones, i.e., stem diameter, $\varnothing$ of V.C. and length of V.B. were greatly decreased with salinity stress level 9000 $\mathrm{mgl}^{-1}$ compared with control. In other words, moringa plant irrigated with tap water (control) manifested the best results as it exceeded that of salinity $9000 \mathrm{mgl}^{-1}$ in terms of most studied anatomical features. Generally, stem anatomical features decreased with salinity at $9000 \mathrm{mgl}^{-1}$ which recorded lower values in the studied anatomical characteristics when compared with control.

\subsection{Response to foliar application nanoparticle treatments}

Data in same Table (3) and Figure (3) obviously indicate the effect of applied nutrients nanoparticle individually treatments i.e., zinc, iron and copper oxide nanoparticles at $150 \mathrm{mgl}^{-1}$ of each as well as Silica nanoparticles at $60 \mathrm{mgl}^{-1}$ on different anatomical features of moringa stem . In this respect, most of these applied treatments have a positively impact on studied histological characteristics of stem i.e., stem diameter, $\varnothing$ of V.C. and length of V.B. when compared with control. Also, the obtained results clearly show that 
Moringa oleifera $\mathrm{L}$. plants treated with zinc foliar spray followed by iron and copper oxide nanoparticles at $150 \mathrm{mgl}^{-1}$ of each appeared to be the most effective treatments compared with silica nanoparticles at $60 \mathrm{mgl}^{-1}$ and control.

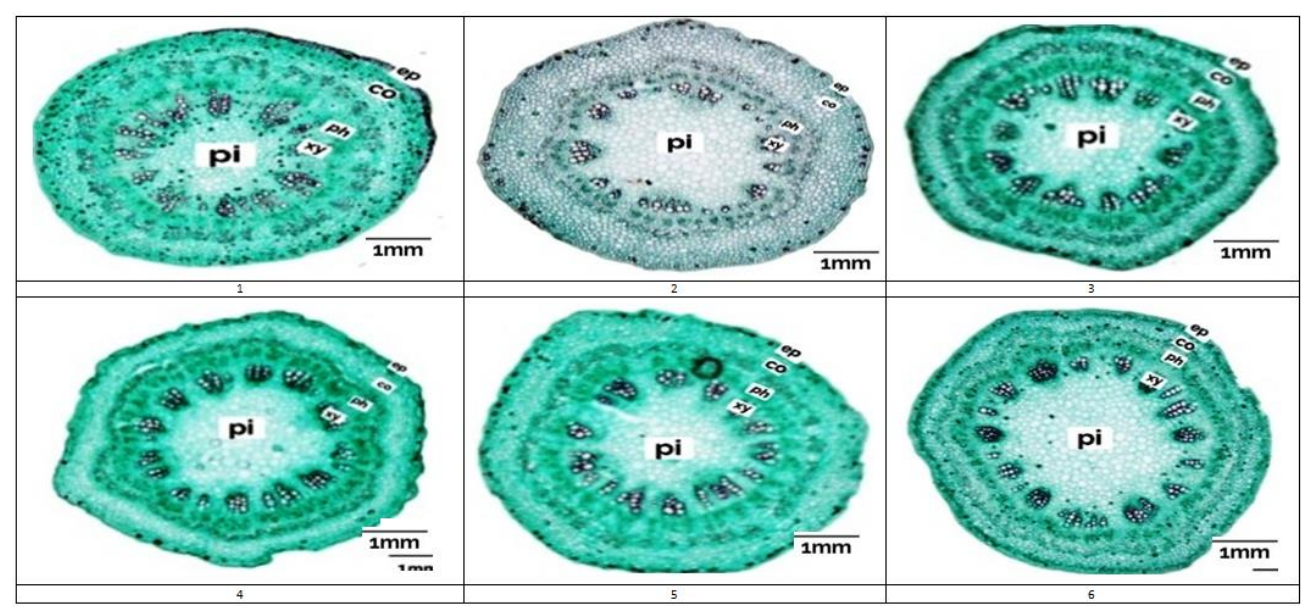

Figure (3): Transverse sections (X 12.5) of moringa stem at 50 days as affected by different applied treatments. Where: (1): Control, (2): Salinity $9000 \mathrm{mgl}^{-1}$, (3): Zinc $150 \mathrm{mgl}^{-1}$, (4): Iron $150 \mathrm{mgl}^{-1}$, (5): Copper $150 \mathrm{mgl}^{-1}$, (6): Silica $60 \mathrm{mgl}^{-1}$, ep= epidermis, co= cortex tissue, $\mathrm{ph}=$ phloem tissue, $x y=$ xylem tissue, pi= pith tissue.

Foliar application treatments with Zinc, Iron, Copper oxide nanoparticles and Silica nanoparticles recorded highly values of the stem studied anatomical characteristics, respectively. Our results are in harmony with those reported by El Feky et al. (2013) found that epidermis cells of the control were similar in shape and size, while the epidermal cells of the NP-treated leaves became larger in size and reached a maximum size when 3 $\mathrm{mgl}^{-1} \mathrm{Fe} 3 \mathrm{O} 4$ Nano Particles NPS foliar spray was used. In addition, the thickness of mesophyll tissue, which is specialized photosynthetic tissue that contains chloroplasts in palisade and spongy parenchyma tissue, was greater in $\mathrm{Fe} 3 \mathrm{O} 4$ treated leaves compared to control leaves. Abou-Shlell (2017) indicated that foliar application of moringa plant with Lithovit (Fe-nano particles) at $500 \mathrm{mgl}^{-1}$ increased moringa stem and leaf anatomical measurements i.e., stem diameter, phloem and xylem tissues thickness as well as vessel diameter.

\subsection{Response to salinity stress and nanoparticle interaction}

As shown in same Table (3) and Figure (4) Data indicate the effect of different applied nutrient nanoparticle treatments upon alleviating the adverse effects of salinity stress on different studied 
anatomical features of Moringa oleifera L. stem. In this respect, most of the applied foliar spray treatments have a positively impact on most histological characteristics of moringa stem i.e., stem diameter and $\varnothing$ of V.C. as well as length of V.B. under used salinity stress level at $9000 \mathrm{mgl}^{-1}$ compared with the control plant. Also, obtained results clearly show that Moringa oleifera L. plant irrigated with water salinity at $9000 \mathrm{mgl}^{-1}$ in combination with Silica nanoparticles at $60 \mathrm{mgl}^{-1}$ foliar sprays followed by Zinc and Iron oxide nanoparticles at $150 \mathrm{mgl}^{-1}$ of each treatment appeared to be the most effective treatments upon alleviating the adverse effects of the highest salinity stress. In general, the stimulatory effects of applied treatments upon the anatomy features of treated plants could be attributed to the effect upon cambium activity. Increment of cambium activity could mainly attributed to the increase of endogenous hormones level especially cytokinins and auxins (Abd El-Aal and Eid, 2017; Sotiropoulos et al., 2002). Generally, the obtained results clearly indicate that different applied nutrient nanoparticle treatments play a defensive protective role against adverse effects of highest salinity stress level via its regulatory functions.

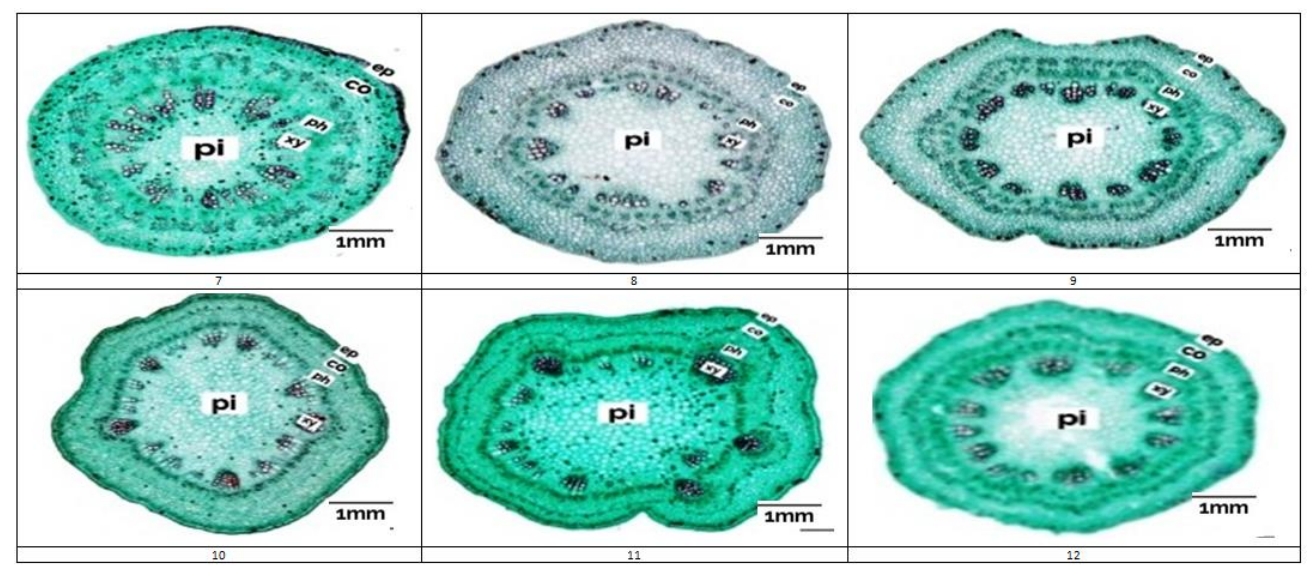

Figure (4): Transverse sections (X 12.5) of moringa stem at 50 days as affected by different applied treatments. Where: (7): Control, (8): Salinity $9000 \mathrm{mgl}^{-1}$, (9): Salinity with zinc $150 \mathrm{mgl}^{-1}$, (10): Salinity with iron $150 \mathrm{mgl}^{-1},(11)$ : Salinity with copper $150 \mathrm{mgl}^{-1},(12)$ : Salinity with silica $60 \mathrm{mgl}^{-1}$, $\mathrm{ep}=$ epidermis, $\mathrm{co}=$ cortex tissue, $\mathrm{ph}=$ phloem tissue, $\mathrm{xy}=\mathrm{xylem}$ tissue, $\mathrm{p}=$ pith tissue.

\subsubsection{Effect of applied treatments on anatomical characteristics of moringa leaflet}

\subsection{Response to salinity stress}

Data presented in Table (3) and Figure (5) illustrate that studied histological features of moringa leaflet also behaved as the same as root and stem anatomical features. Since, the treatment of salinity 
stress level at $9000 \mathrm{mgl}^{-1}$ decreased most of Moringa oleifera L. leaflet histological features compared with control. High reductions were recorded in many anatomical features thickness with salinity stress level which negatively affected on studied anatomical characteristics. Among these anatomical features were the most important ones i.e., upper and lower epidermis thickness as well as mesophyll tissues (palisade and spongy tissues) were decreased when compared with the control. In other words, Moringa oleifera L. plant of control recorded best results as it exceeded that of salinity stress level at $9000 \mathrm{mgl}^{-1}$ in terms of most studied anatomical features. In general, the above mentioned results clearly indicate that salinity stress level of irrigation water had opposite effect on those leaflet anatomical characteristics of Moringa oleifera $\mathrm{L}$. plant. The obtained results are in harmony with those reported by Child et al. (2003), Abdel and Al-Rawi (2011) and El-Afry et al. (2012).

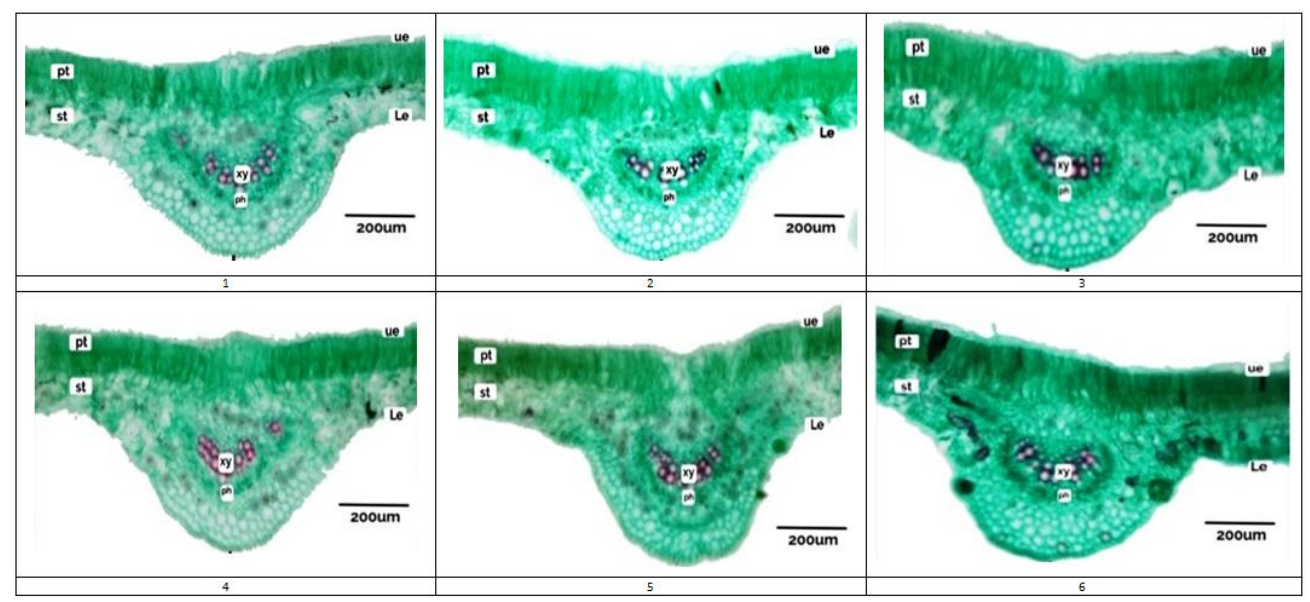

Figure (5): Transverse sections (X 12.5) of moringa leaflet at 50 days as affected by different applied treatments. Where: (1): Control, (2): Salinity $9000 \mathrm{mgl}^{-1}$, (3): Zinc $150 \mathrm{mgl}^{-1}$, (4): Iron $150 \mathrm{mgl}^{-1}$, (5): Copper $150 \mathrm{mgl}^{-1},(6)$ : Silica $60 \mathrm{mgl}^{-1}$, ue= upper epidermis, $\mathrm{pt}=$ palisade tissue, $\mathrm{st}=$ spongy tissue, le= lower epidermis, $\mathrm{ph}=$ phloem tissue, $\mathrm{xy}=\mathrm{xylem}$ tissue.

\subsection{Response to foliar application nanoparticle treatments}

Data presented in Table (3) and Figure (5) clearly show the simulative effect of different applied nanoparticle individually treatments on some anatomical features of Moringa oleifera
L. leaflet compared with the control plant. Same data clear the simulative effect of foliar spray with nanoparticle treatments which led to maintain the highest values of studied histological features of moringa leaflet i.e., upper and lower epidermis thickness as well as mesophyll tissues (palisade and spongy 
tissues) compared with control. In highest values as exceeded that of other general, foliar application with zinc foliar applications with copper and silica followed by iron oxide nanoparticles at nanoparticle treatments in terms of the $150 \mathrm{mgl}^{-1}$ of each treatment recorded the most studied anatomical features.

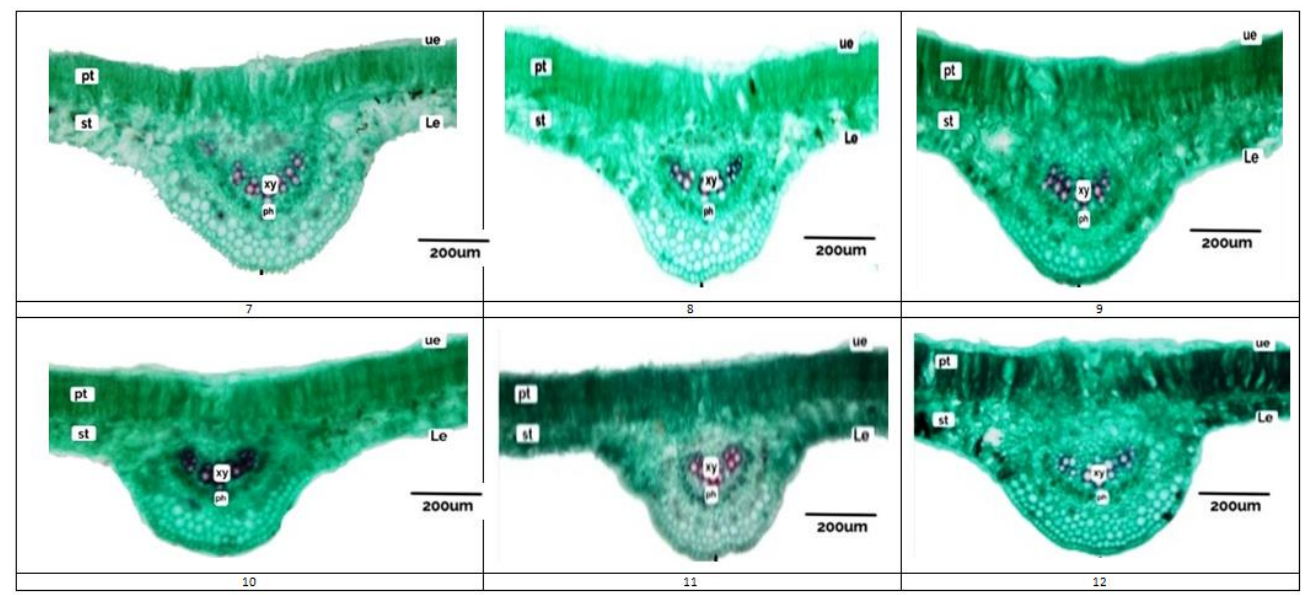

Figure (6): Transverse sections (X 12.5) of moringa leaflet at 50 days as affected by different applied treatments. Where: (7): Control, (8): Salinity $9000 \mathrm{mgl}^{-1}$, (9): Salinity with zinc $150 \mathrm{mgl}^{-1}$, (10): Salinity with iron $150 \mathrm{mgl}^{-1},(11)$ : Salinity with copper $150 \mathrm{mgl}^{-1},(12)$ : Salinity with silica $60 \mathrm{mgl}^{-1}$, $\mathrm{ue}=$ upper epidermis, $\mathrm{pt}=$ palisade tissue, $\mathrm{st}=$ spongy tissue, $\mathrm{le}=$ Lower epidermis, $\mathrm{ph}=\mathrm{phloem}$ tissue, $\mathrm{xy}=$ xylem tissue.

\subsection{Response to salinity stress and nanoparticle interactions}

Data presented in Table (3) and Figure (6) clearly indicate the simulative effect of different applied nanoparticle treatments upon alleviating the adverse effects of salinity stress on some anatomical features of Moringa oleifera L. leaflet. Once again, same data clear the positive effect of foliar applications with zinc, iron, copper and silica nanoparticle treatments which recorded the highest values of moringa leaflet histological features i.e., upper and lower epidermis thickness as well as mesophyll tissues (palisade and spongy tissues) under salinity stress compared with the control. Also, the obtained results clearly show that moringa plant irrigated with water salinity at $9000 \mathrm{mgl}^{-1}$ in combination with Silica nanoparticles at $60 \mathrm{mgl}^{-1}$ foliar sprays followed by Zinc oxide nanoparticles at $150 \mathrm{mgl}^{-1}$ appeared to be the most effective treatments upon alleviating the adverse effects of the salinity stress compared with other treatments and control. Finally we can conclude with, improving moringa plant tolerance to such salinity stress level can be achieved by application of some simulative substances to enhance its growth and maximizing plant yield. 


\section{References}

Abd El-Aal, M. M. M. and Eid, R. S. M. (2017), "Optimizing growth, seed yield and quality of soybean (Glycine max L.) plant using growth substances", Asian Research Journal of Agriculture, Vol. 6 No. 3 pp. 1-19.

Abd El-Aal, M. M., El-Anany, A. M. and Rizk, S. M. (2019), "Rationalization of water consumption for taro plant through the rationing of irrigation and expand the plant ability to resist stress conditions", International Journal of Plant \& Soil Sciences, Vol.29, No.4, pp. 1-23.

Abdel, C. G. and Al-Rawi, I. M. T. (2011), "Anatomical alteration in response to irrigation and water stress in some legume crops". American Journal of Experimental Agricultural, Vol. 1 No.4, pp. 231264.

Abdelhamid, M. T., Shokr, M. M. B. and Bekheta, M. A. (2010), "Growth, root characteristics, and leaf nutrients accumulation of four faba bean (Vicia faba L.) cultivars differing in their broomrape tolerance and the soil properties in relation to salinity", Communications in Soil Sciences and Plant Analysis, Vol. 41 No.22, pp. 2713-2728.

Abdull Razis, A. F., Ibrahim, M. D. and Kntayya, S. B. (2014), "Health Benefits of Moringa oleifera", Asian Pacific-Basin Journal Cancer
Prevention, Vol. 15, No. 20, pp. 8571-8576.

Abou El-Nasr, M. K., El-Hennawy, H. M., El-Kereamy, A. M. H., Abou ElYazied, A. and Salah Eldin, T. A. (2015), "Effect of Magnetite Nanoparticles (Fe3O4) as Nutritive Supplement on Pear Saplings", Middle East Journal Applied. Sciences, Vol. 5 No. 3, pp. 777-785.

Abou-Shlell, M. K. (2017), Botanical studies on Moringa plant, MS Thesis, Agricultural Botany Department, Faculty of Agriculture, Moshtohor, Banha University, USA, pp. 154.

Akram, M., Akhtar, S., Javed, I. H., Wahid, A. and Rasul, E. (2002), "Anatomical attributes of different wheat (Triticum aestivum) accessions/varieties to $\mathrm{NaCl}$ salinity", International Journal of Agriculture and Biology, Vol. 4, pp. 166-168.

An, P., Inanaga, S., Schimizu, L. X. and Tanimoto, H. E. (2003), "Root characteristics in salt tolerance", Root Research, Vol. 12, pp. 125132.

Asad, A. and Rafique, R. (2000), "Effect of zinc, copper, manganese and boron on the yield and yield components of wheat crop in Tehsil Peshawar". Pakistan Journal Biological Sciences, Vol. 3, pp. 1615-1620.

Asin, L., Alegre, S. and Montserrat, R. 
(2007), "Effect of paclobutrazol, prohexadione-ca, deficit irrigation, summer pruning and root pruning on shoot growth, yield and return bloom in "Blanquilla" pear orchard", Sciences Horticulture, Vol. 113 No. 2, pp. 135-142.

Awang, Y. B., Atherton, J. G. and Taylor, A. J. (1993), "Salinity effects on strawberry plants in Rockwool. Growth and leaf water relations", Journal Horticultural Sciences, Vol. 68, pp.783-790.

Awasthi, O. P. and Pathak, R. K. (1999), "Effect of salinity levels on survival and anatomy of four scion cultivars budded on Indian jujube", Horticultural of Journal, Vol. 12, pp. 53-59.

Ayers, R. S. and Westcot, D. W. (1985), Water quality for agriculture, FAO Irrigation and Drainage Paper 29 (Rev. 1), Food and Agriculture Organization (FAO) of the United Nations, Rome, Italy.

Baddaruddin, M., Reynolds, M. P. and Ageeb, O. A. A. (1999), "Wheat management in warm environment: effect of organic and inorganic fertilizers, irrigation frequency and mulching", Agronomy Journal, Vol. 91, pp. 975-981.

Belda, R. M. and Ho, L. C. (1993), "Salinity effects on the network of vascular bundles during tomato fruit development", Journal Horticultural Sciences, Vol. 68 pp. 557-564.
Bellaire, B. A., Carmody, J., Braud, J., Gosset, D. R., Banks, S. W. and Cran Lucas, M. (2000), "Involvement of abscisic aciddependent and independent pathways in the upregulation of antioxidant enzyme activity during $\mathrm{NaCl}$ stress in cotton callus tissue", Free Radiation Research, Vol. 33, pp. 531-545.

Botti, C., Palzkill, D., Munoz, D. and Prat, L. (1998), "Morphological and anatomical characterization of six jojoba clones at saline and nonsaline sites", Indian Crop Production, Vol. 9, pp. 53-62.

Brugnoli, E. and Lauteri, M. (1991), "Effect of salinity on stomatal conductance, photosynthetic capacity, and carbon isotope discrimination of salt tolerant (Gossypium hirsutum L.) and saltsensitive (Phaseolus vulgaris $\mathrm{L}$.) $\mathrm{C}_{3}$ nonhalophytes", Environmental and Stress Physiology, Vol. 95 No. 2, pp. 628-635.

Cakmak, I. and Marschner, H. (1988), "Zinc- dependent changes in ESR signals, NADPH oxidase and plasma membrane permeability in cotton roots", Physiologia Plantarum, Vol. 73, pp. 132-186.

Chaparzadeh, N., D'Amico, M. L., Khavari-Nejad, R. A. and Izzo, F. (2004), "Antioxidative response of Calandula officinalis under salinity conditions", Plant Physiology and Biochemistry, Vol. 42, pp. 695-701. 
Child, R. D., Summers, J. E., Babij, J., Farrent, J. W. and Bruce, D. M. (2003), "Increased resistance to pod chatter is associated with changes in the vascular structure in pods of a resynthesized Brassica napus line", Journal Experimental Botany, Vol. 54, pp. 1919-1930.

Curtis, P. S. and Lauchli, A. (1987), "The effect of moderate salt stress on leaf anatomy in Hibiscus cannabinus (Kenaf) and its relation to leaf area", American Journal of Botany, Vol. 74, pp. 538-542.

Dajic, Z. (2006), "Salt stress", in Physiology and Molecular Biology of Salt Tolerance in Plant, Madhava Rao K.V., Raghavendra A.S., and Janardhan Reddy K. Eds., SpringerVerlag, Dordrecht, Holland, pp. 4199.

Dionisio-Sese, M. L. and Tobita, (2000), "Effects of salinity on sodium content and photosynthetic responses of rice seedlings differing in salt tolerance", Journal Plant Physiology, Vol. 157, pp. 54-58.

Dorais, M. Papadopoulos, A. P. and Gosselin, A. (2001), "Green house tomato fruit quality", Horticultural Revista Reviews, Vol. 26, pp. 239319.

El-Afry, M. M., Mohamed, F. E., Elsayed, B. A. and Metwaly, M. S. M. (2012), "Anatomical studies on drought-stressed wheat plants (Triticum aestivum L.) Treated with some bacterial strains", Acta
Biological Szeged, Vol. 56, No. 2, pp. 165-174.

Elfeky, S. A., Mohammed, M. A. Khater, M. S., Osman, Y. A. H. and Elsherbini, E. (2013), "Effect of magnetite Nano-Fertilizer on Growth and yield of Ocimum basilicum L". International Journal of Indian Medical, Vol. 46 No. 3, pp. 1286-1293.

Emadian, S. F. and Newton, R. J. (1989), "Growth enhancement of Loblolly pine (Pius taeda L) seedlings by silicon", Journal of Plant Physiology, Vol. 134, pp. 98-103.

Etesamy, H. and Jeong, B. R. (2018), "Silicon (Si): Review and future prospects on the action mechanisms in alleviating biotic and a biotic stresses in plants", Eco toxicology and Environmental Safety, Vol. 147, pp. 881-896.

Fahey, J. W. (2005), "Moringa oleifera: A review of the medical evidence for its nutritional, therapeutic, and prophylactic properties", Trees for Life Journal, Vol. 1, pp. 1-5.

Gharib, A. A. and Hanafy Ahmed, A. H. (2005), "Response of pea (Pisum sativum, L.) to foliar application of putrescine, glucose, foliafeed D and silicon", Journal of Agricultural Science, Mansoura University, Vol. 30 No.12, pp.7563-7579.

Gomaa, A. M. El-Mesiry, T. and Rady, M. (2008), "The potential impact of biofertilization, antioxidant and 
micronutrients application in reducing salinity stress on two wheat cultivars", Australian Journal of Basic and Applied Sciences, Vol. 2 No. 3, pp. 575- 582 .

Gomez K. A. and Gomez A. A. (1984), Statistical procedures for agricultural research, $2^{\text {nd }}$ ed., John Wiley and Sons, USA.

Greenway, H. and Munns, R. (1980), "Mechanism of salt tolerance in nonhalophytes", Annuual Revista Reviews Plant Physiological, Vol. 31, pp. 149-190.

Grossmann, K., König-Kranz, S. and Kwiatkowski, J. (1994), "Phytohormonal changes in intact shoots of wheat and oilseed rape treated with the acylcyclohexanedione growth retardant prohexadione calcium", Physiologia Plantarum, Vol. 90 No. 1, pp. 139-143.

$\mathrm{Hu}$, Y. and Schmidhalter, U. (2001), "Reduced cellular crosssectional area in the leaf elongation zone of wheat causes a decrease in dry weight deposition under saline conditions", Australian Journal Plant Physiology, Vol. 28, pp.165170.

Hu, Y., Burucs, Z., Tucher, Von, S. and Schmidhalter, U. (2007), "Shortterm effects of drought and salinity on mineral nutrient distribution along growing leaves of maize seedlings", Environmental and Experimental Botany, Vol. 60, pp.
268-275.

Johanson, D. V. (1940), Plant microtechnique, McGraw-Hill Book Company, Inc., London, New York, USA, pp. 27-154.

Jonas, O. A., Pereyra, M. C., Cebeza, C., Golberg, A. D. and Ledent, J. F. (1992), "Recovery of nitrate reductase activity in wheat leaves after a period of severe water stress", Cereal Research Communications, Vol. 20, pp. 13-18.

Junghans, U., Polle, A., Duchting, P., Weiller, E., Kuhlman, B., Gruber, F. and Teichmann, T. (2006), "Adaptation to high salinity in poplar involves changes in xylem anatomy and auxin physiology", Plant Cell Environmental, Vol. 29, pp. 1519-1531.

Kanwal, H., Hameed, M., Nawaz, T., Ahmad, M. S. A. and Younis, A. (2012), "Structural adaptations for adaptability in some exotic and naturalized species of Agavaceae", Pakistan Journal of Botany, Vol. 44 pp. 129-134.

Kaya, C. and Higgs, D. (2002), "Response of tomato (Lycopersicon esculentum L.) cultivars to foliar application of zinc in sand culture at low zinc", Sciences Horticulture, Vol. 93 pp. 53- 64.

Khosravinejad, H. F. R. and Farboondia, T. (2008), "Effect of salinity on photosynthetic pigments, respiration and water content in barley 
varieties", Pakistan Journal Biological Sciences, Vol. 11, pp. 2438-2442.

Kiliç, S., Çavuşoğlu, K. and Kabar, K. (2007), "Effects of 24epibrassinolide on salinity stress induced inhibition of seed germination, seedling growth and leaf anatomy of barley", Süleyman Demirel University Faculty of Arts and Science Journal of Science, Vol. 2 pp. 41-52.

Laing, M., and Adandonon, A., (2005), Silicon and insect managementreview, In: GH Korndörfer (Ed.), Proceedings of the III Silicon in Agriculture Conference, Federal University of Uberlândia, Minas Gerais, Brazil, pp. 41-50.

Lu, J., Zhao, H., Chen, J., Fan, W., Dong, J., Kong, W., Sun, J., Cao, Y. and Cai, G. (2007), "Evolution of phenolic compounds and antioxidant activity during malting", Journal of Agricultural and Food Chemistry, Vol. 55 No. 26, pp. 10994-11001.

Ludders P.C. and Kaminski, V. (1991), "Einflub von $\mathrm{NaCl}$ auf die stomataund blatthaardichte bei feigen in unterschiedlicher luftfeuchte", Mitteilungen Klosterneuburg, Vol. 41, pp.76-78.

Ma, J. F. (2004), "Role of silicon in enhancing the resistance of plants to biotic and a biotic stress", Soil Sciences of Plant Nutrition, Vol. 50. No.1, pp. 11-18.

Marschner, H. (1995), Mineral nutrition of higher plants, $2^{\text {nd }}$ ed., Academic Press, Harcourt Brace and Company, New York, USA.

McCauley, A., Jones, C. and Jacobsen, J. (2009), Plant nutrient functions and deficiency and toxicity symptoms, Nutrient management module No. 9, A self-study course from the MSU Extension Service Continuing Education Series, Montana State University, USA, pp. 16.

Mckersie, B. D., Bowtey, S. R., Harjanto, E. and Leprice, O. (1996), "Waterdeficit tolerance over-expressing superoxide dismutase", Plant Physiology, Vol. 111, pp. 13211326.

Mekkdad, A. A. A. (2017), "Response of peanut nitrogen fertilizer levels and foliar zinc spraying rates in newly reclaimed sandy soils", Journal plant production Mansoura University, Vol. 8 No.2, pp. 153159.

Mishra, S. P., Singh, P. and Singh, S. (2012), "Processing of Moringa oleifera leaves for human consumption", Bull Environmental Pharmacology Life Sciences, Vol. 2 No.1, pp.28-31.

Munns, R. (2003), "Physiological processes limiting plant growth in saline soils. Some dogmas and hypotheses", Plant Cell and Environmental, Vol. 16, pp. 15-24.

Nawaz, T., Hameed, M., Ashraf, M., Ahmad, F., Ahmad, M. S. A., 
Hussain, M., Ahmad, I., Younis, A. and Ahmad, K. S. (2012a), "Diversity and conservation status of economically important flora of the salt range", Pakistan Journal of Botany, Vol. 44, pp. 203-211.

Nawaz, T., Hameed, M., Nisa, W., Ahmad, M. S. A., Younis, A. and Kanwal, H. (2012b), "Comparative anatomy of root and stem of some native and exotic Asparagus L. species", Pakistan Journal of Botany, Vol. 44, pp. 153-158.

Nawazish, S., Hameed, M. and Naurin, S. (2006), "Leaf anatomical adaptations of Cenchrus ciliaris L. from the salt range, Pakistan against drought stress", Pakistan Journal of Botany, Vol. 38, pp. 1723-1730.

Neumann, P. (1995), "Inhibition of root growth by salinity stress: toxicity or an adaptive biophysical response", Structure and function of roots, Baluska $\mathrm{F}$, Ciamporová M, Gasparikova MO, Barlow PW, eds., Academic Kluwer Publishers, Dordrecht, pp. 299-304.

Osman, H. E. and Abohassan, A. A. (2012), "Morphological and Analytical characterization of Moringa peregrine populations in Western Saudi Arabia", International Journal of Theoretical and Applied Sciences, Vol. 4 No.2, pp.174-184.

Pasternak, D. (1987), "Salt tolerance and crop production: a comprehensive approach", Annual Revista Reviews Phytopathology, Vol. 25, pp. 271291.

Pimmongkol, A. S., Terapongtanakhon and Udonsirichakhon, K. (2002), Anatomy of salt-and nonsalt-tolerant rice treated with $\mathrm{NaCl}, 28^{\text {th }}$ Congress, Science and Technology of Thailand, Bangkok, Thailand.

Prasad, T., Sudhakar, P., Sreenivasulu, Y., Latha, P., Munaswamy, V., Reddy, K. R. (2012), "Effect of nanoscale zinc oxide particles on the germination, growth and yield of peanut", Journal Plant Nutrition, Vol. 35 No .6, pp.905-927.

Price, A. H. and Hendry, G. A. F. (1991), "Iron catalyzed oxygen radical formation and its possible contribution to drought damage in nine native grasses and three cereals", Plant Cell Environmental, Vol. 14, pp. 477-484.

Quary, F. X., Leenhardt, F. and Remesy, C. (2006), "Genetic variability and stability of grain $\mathrm{Mg}, \mathrm{Zn}$ and $\mathrm{Fe}$ concentration in bread wheat", European Journal of Agronomy, Vol. 25, No.2, pp. $17-185$.

Raafat, A., Habib, S. A., El-Shami, I. Z. and El-Antably, H. M. (1991), "The effect of salinity on the anatomical features of tomato plants", Annual Agricultural Sciences, Vol. 36, pp. 307-321.

Reinhardt, D. H., Rost, T. L. (1995), "Salinity accelerates endodermal 
development and induces an exodermis in cotton seedlings roots", Environmental and experimental Botany, Vol. 35, pp. 563-574.

Remero Aranda, M. R., Jurado, O. and Cuartero, J. (2006), "Silicon alleviates the deleterious salt effect on tomato plant growth by improving plant water status", Journal Plant physiology, Vol. 163 No. 8, pp.847-855.

Romheld, V. and Marschner, H. (1991), "Genotypic differences among graminaceous species in release of phytosiderophores and uptake of iron of phytosiderophores", Plant Soil, Vol.123, pp. 147-153.

Sadasivam, S. and Manickam, A. (1997), Biochemical Methods, $2^{\text {nd }}$ edition, New Age International Publishers Limited, New Delhi, India.

Saint-Cricq De Gaulejac, N., Provost, C. and Vivas, N. (1999), "Comparative study of polyphenol scavenging activities assessed by different methods", Journal Agricultural Food Chemistry, Vol. 47 No. 2, pp. 425-431.

Sakr, M. T., Heba, M. A., Marouah, I. A. and Moustafa, A. A. A. (2013), "Alleviating the harmful effect of salinity stress on soybean plants by using some promoters", Journal Plant Production Mansoura University, Vol. 4, pp. 205-218.

Sass, J. E. (1951), Botanical microtechnique, Iowa state college press, Ames, Iowa,USA, pp. 228.

Sayed, S. M., Abd El-Dayem, H. M., ElDesouky, S. A., Khedr, Z. M. and Samy, M. M. (2018), Effect of silicon and algae extract foliar application on growth and early yield of globe artichoke plants, $4^{\text {th }}$ International Conference on Biotechnology Applications in Agriculture (ICBAA), Benha University, Moshtohor and Hurghada, Egypt, pp. 207-214.

Sedghi, M., Hadi, M. and Toluie, S. G. (2013), "Effect of nano zinc oxide on the germination of soybean seeds under drought stress", Annals of West University of Timisoara, ser. Biology, Vol. XVI(2), pp.73-78.

Shkolnik, M. Y. (1984), "Trace Elements in Plants. Elsevier, Amsterdam.

Smirnoff, N. (1998), "The role of active oxygen in the response of plants to water deficit and desiccation", New Phytologist, Vol. 125, pp. 27-58.

Sotiropoulos, T. E., Therios, I. N., Dimassi, K. N., Bosabalidis, A. and Kofidis, G. (2002), "Nutritional status, growth, $\mathrm{CO}_{2}$ assimilation and leaf anatomical responses in two kiwi fruit species under boron toxicity", Journal of Plant Nutrition, Vol. 25 No. 6, pp. 1249-1261.

Tanaka, K., Tankeuchi, E., Kubo, A., Sakaki, T., Haraguch, K. and Kawamura, Y. (1994), "Two immunologically different 
isoenzyme of ascorbate peroxidase Younis, A., Riaz, A., Ahmed, I., from spinach leaves", Archives of Siddique, M. I., Tariq, U., Hameed, Biochemistry and Biophysics, Vol. M. and Nadeem, M. (2014), 286, pp. 371-375.

Wilson, C., Liu, X., Lesch, S. M. and Suarez, D. L. (2006), "Growth response of major USA cowpea cultivars. I. Biomass accumulation and salt tolerance", Horticultural Sciences, Vol. 41, pp. 225-230.

Yildirim, B., Yaser, F., Ozpay, T., Ozpay, D. T., Turkozu, D. Terziodlu, O. and Tamkoc, A. (2008), "Variations in response to salt stress among field pea genotypes (Pisum sativum sp. Arvense L.)", Journal Animals Veterinary Advances, Vol. 7, pp. 907-910. 